\title{
Quantum walks: The mean first detected transition time
}

\author{
Q. Liu $\odot,{ }^{1,2,{ }^{*}}$ R. Yin $\odot,{ }^{1}$ K. Ziegler $\odot,{ }^{3}$ and E. Barkai ${ }^{1}$ \\ ${ }^{1}$ Department of Physics, Institute of Nanotechnology and Advanced Materials, Bar-Ilan University, Ramat-Gan 52900, Israel \\ ${ }^{2}$ School of Physics, Taishan College, Shandong University, Jinan 250100, China \\ ${ }^{3}$ Institut für Physik, Universität Augsburg, D-86135 Augsburg, Germany
}

(Received 2 January 2020; accepted 15 June 2020; published 21 July 2020)

\begin{abstract}
We consider the quantum first detection problem for a particle evolving on a graph under repeated projective measurements with fixed rate $1 / \tau$. A general formula for the mean first detected transition time is obtained for a quantum walk in a finite-dimensional Hilbert space where the initial state $\left|\psi_{\text {in }}\right\rangle$ of the walker is orthogonal to the detected state $\left|\psi_{\mathrm{d}}\right\rangle$. We focus on diverging mean transition times, where the total detection probability exhibits a discontinuous drop of its value by mapping the problem onto a theory of fields of classical charges located on the unit disk. Close to the critical parameters of the model, we find simple expressions describing the blow-up of the mean transition time. Using previous results on the fluctuations of the return time, corresponding to $\left|\psi_{\text {in }}\right\rangle=\left|\psi_{\mathrm{d}}\right\rangle$, we find close to these critical parameters that the mean transition time is proportional to the fluctuations of the return time, an expression reminiscent of the Einstein relation.
\end{abstract}

DOI: 10.1103/PhysRevResearch.2.033113

\section{INTRODUCTION}

A closed quantum system is prepared in some initial state and evolves unitarily over time. Our aim is to monitor the evolution of this system by repeated projective measurements until a certain state is detected for the first time. A corresponding simple classical example [1,2] would be to take a picture of a rare animal in the wilderness. For this purpose a remote camera takes pictures at a fixed rate, and the camera's software checks immediately whether the rare animal is on the last picture. Once the animal is caught on the last snapshot the process stops. It is obvious that we may miss the first appearance of the animal in the process. But when we continue long enough we might be lucky. The theoretical question is, what would be "long enough" to detect the animal at a given measurement rate? This is the problem of the classical first passage time that has been a well-studied field of research $[1,2]$. More recently, substantial work has been devoted to the analysis of the corresponding quantum problem, both theoretically as well as experimentally. Instead of a picture based on classical random walks which models the dynamics of a particle before arriving on a target, in quantum search one uses quantum walks instead [3-8]. To investigate the quantum first detection problem one adds to the quantum walk a detection protocol, where the latter affects and modifies the unitary evolution. The quantum first detection problem has been considered in detail [9-20] as part of a wider investigation of a unitary evolution pierced by measurements [21-28].

\footnotetext{
*Corresponding author: qcliu.ac@gmail.com

Published by the American Physical Society under the terms of the Creative Commons Attribution 4.0 International license. Further distribution of this work must maintain attribution to the author(s) and the published article's title, journal citation, and DOI.
}

Recent realizations of quantum systems with a few electrons, ions, or neutral atoms [29-32] have revealed new opportunities to study the above-mentioned monitored evolution. To support this development it would be useful to establish a general theory which can be adapted to specific physical systems. In particular, this theory could lead to advances in physical realizations of quantum search algorithms [9,33-36]. As a contribution to this effort we present an approach for a quantitative analysis of the monitored transition to a specific state of a quantum system which is defined by a time-independent Hamiltonian. The only restriction for the latter is the assumption that it acts on a finite-dimensional Hilbert space. This implies that typical realizations are a single or a few particles on a discrete (tight-binding) structure or spin systems on a lattice. This line of research was considered by Krovi and Brun [10-12], who showed that even for small systems the hitting time can be infinite. These authors discussed the specific example of a quantum walk on a hypercube in detail and demonstrated that there is either a speed-up or a slow down in comparison with the classical random walk, depending on the conditions of the initial and the final state as well as on the evolution operator. In the following we will first analyze some general relations between the spectrum and the spectral weights of the underlying Hamiltonian and the mean first detected transition (FDT) time. In the second part we will study some specific models for a quantum particle on a finite graph.

In our approach we employ stroboscopic sampling, which is flexible due to a tunable sampling rate $1 / \tau$. On the other hand, this type of sampling is accessible by a generating function technique [18]. More importantly, the monitored quantum evolution can be mapped to a classical charge theory. For instance, we will show how the mean FDT time is related to the stationary points of a set of classical charges positioned on the unit circle in the complex plane with locations $\exp \left(i E_{j} \tau\right)$, where $E_{j}$ is an energy level of the underlying Hamiltonian. 
This charge picture was previously promoted in the context of the return problem [13], where the systems starts from an initial state and returns to this state after some time. It allows us to develop an intuitive picture of the quantum problem, based on classical electrostatics. Here we will demonstrate that this mapping between quantum and classical physics is also be applicable to the transition problem, where the systems starts in some initial state orthogonal to the detected state. On the other hand, return and transition mean detection times properties exhibit a vastly different behavior. For instance, in the return problem the mean return time is quantized due to a topological invariant $[13,37]$. Thus, the average return time depends only on the effective dimensionality of the underlying Hilbert space, which is given by the number of distinct energy levels with nonzero overlap with the target space, in units of the time step $\tau$. In contrast, the mean FDT time is very sensitive, for instance, to the sampling rate $1 / \tau$. If we sample too fast, then the transition cannot be detected at all due to the Zeno effect [16-18,38]. This implies that there exist special sampling times that are optimal, in the sense that the detection time attains a minimum.

The paper is organized as follows: In Sec. II we define our model and the degeneracies caused by the sampling time $\tau$. Then we derive our first main result the mean FDT time in Sec. III. We find the general relation of the transition time and return time fluctuations in Sec. IV. In Secs. V, VI, VII, and IX we study some characteristic diverging transition times, where special relations for the transition time and the return fluctuations are found. We use some examples to confirm our theory in Sec. VIII. We close the paper with discussions and a summary in Sec. X. More detailed calculations are presented in the appendices.

\section{MODEL AND FORMALISM}

\section{A. Stroboscopic protocol}

We consider a quantum particle prepared in the initial state $\left|\psi_{\text {in }}\right\rangle$ at $t=0$, for instance an electron trapped on a node of the lattice. The evolution of this quantum particle is described by the time-independent Hamiltonian $H$ according to the Schrödinger equation. As an example of the Hamiltonian $H$, consider a one-dimensional tight-binding model in discrete position space with nearest-neighbor hopping:

$$
H=-\gamma \sum_{x=-N}^{N}(|x\rangle\langle x+1|+| x+1\rangle\langle x|) .
$$

However, our general formalism does not rely on a specific Hamiltonian, as long as we are restricted to a finitedimensional Hilbert space.

After every free evolution, we try to detect the particle. In the measurement the detector collapses the wave function at the detected state $\left|\psi_{\mathrm{d}}\right\rangle$ and this is mathematically described by the projection operator $D=\left|\psi_{\mathrm{d}}\right\rangle\left\langle\psi_{\mathrm{d}}\right|$. For simplicity one may assume that $\left|\psi_{\mathrm{d}}\right\rangle$ is yet another localized node state of the graph. We perform the measurements with a discrete time sequence $\tau, 2 \tau, \ldots, n \tau$ until it is successfully detected for the first time. Then the result of the measurements is a string: "no, no, ..., no, yes". In the failed measurements the wave function collapses to zero at the detected state, and
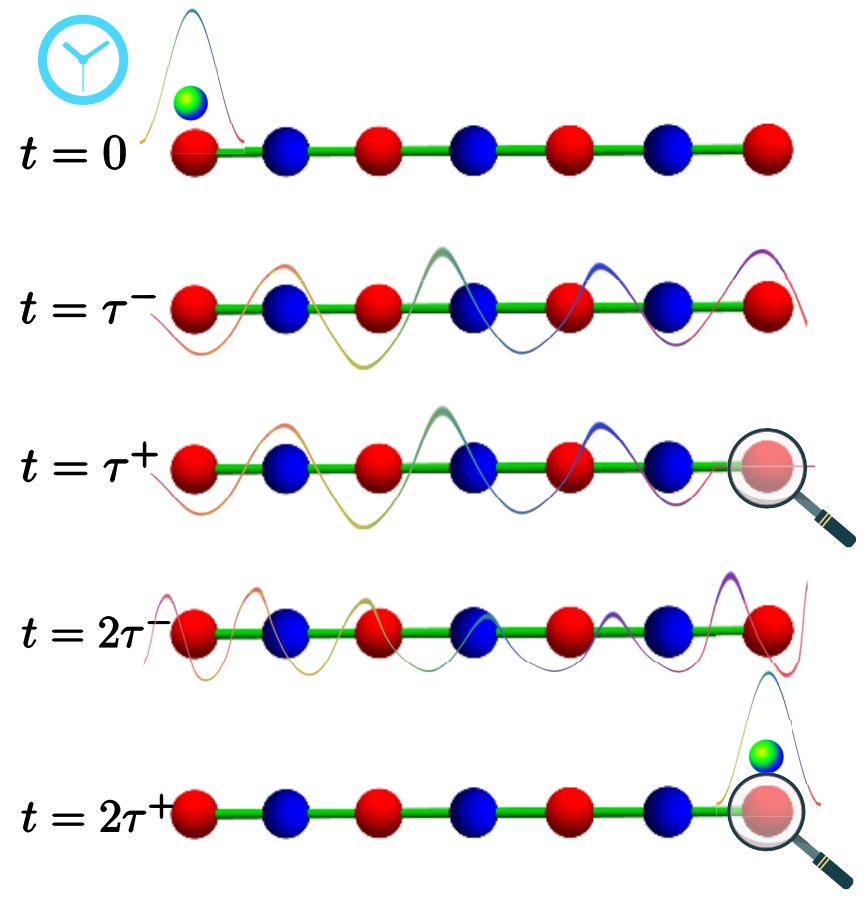

FIG. 1. Schematic plot of the first detected transition problem and the measurement protocol. The quantum particle is prepared at the initial state $\left|\psi_{\text {in }}\right\rangle$ on the lattice at $t=0$ and evolves unitarily in the detection free interval $\tau$. And measurements (the magnifying glass) are performed every $\tau$ units of time. Here $-/+$ means before/after measurement. In the failed attempt $t=\tau$, the detector collapses the wave function at the detected state (collapse theory), namely the amplitude is erased at the detected site. We repeat this process until the quantum particle is successfully detected (for example, here $t=2 \tau$ ). The question is how long it takes to find the quantum particle.

we renormalize the wave function after each failed attempt. The event of detecting the state $\left|\psi_{\mathrm{d}}\right\rangle$ for the first time after $n$ attempts implies that $n-1$ previous attempts failed and this certainly must be taken into consideration. Namely the failed measurements backfire and influence the dynamics by erasing the wave function at the detected state. Finally, the quantum state is detected and the experiment is concluded (see Fig. 1). Hence the first detection time is $t=n \tau$.

The key ingredients of this process are the initial state $\left|\psi_{\text {in }}\right\rangle$ and the detected state $\left|\psi_{\mathrm{d}}\right\rangle$, which characterize this repeated measurements problem. If the initial state is the same as the detected state, namely $\left\langle\psi_{\text {in }} \mid \psi_{\mathrm{d}}\right\rangle=1$, then we call this case the first detected return (FDR) problem, which has been well studied by a series of works $[13,16,37,39,40]$. In the following we investigate the FDT problem, where $\left\langle\psi_{\text {in }} \mid \psi_{\mathrm{d}}\right\rangle=0$. This transition problem describes the transfer of the quantum state from $\left|\psi_{\text {in }}\right\rangle$ to $\left|\psi_{\mathrm{d}}\right\rangle$ in Hilbert space. The time this process takes is of elementary importance. Since the results in each experiment are random, we focus on the expected FDT time $\langle n\rangle \tau$, which gives the average quantum transition time in the presence of the stroboscopic measurements.

\section{B. Brief outlook on the main results}

Before we embark to develop the general formalism, we give a brief outlook. The main goal of this work is to provide 
a method to calculate the mean FDT time for a system with Hamiltonian $H$ under repeated measurement with time step $\tau$. For this purpose we introduce a generating function [18], from which the distribution of FDT times and their moments can be derived. Then we focus on the mean FDT time, which is given at the end of Sec. III. The characteristic parameters of the mean FDT time are solutions of a classical electrostatic equation for whose sources are charges on the unit circle.

Then we study a relation between the mean FDT time and the fluctuations of the FDR time, where the latter was obtained in our previous work [37]. It turns out that near degeneracies of the spectrum of the evolution operator the mean FDT times as well as the fluctuations of the FDR time diverge. These divergences obey an asymptotic relation, similar to the Einstein relation in classical diffusive systems.

The formalism can be applied to physical systems with any finite-dimensional Hilbert space, ranging from two-level systems to correlated many-body systems. A few examples are presented in Secs. VIII A-VIII C and IX.

\section{Generating function}

We denote the wave function after $n-1$ measurements $\left|\psi\left[(n-1) \tau^{+}\right]\right\rangle$. Here $(-/+)$means before/after measurement. By the definition, during the time interval $\tau$ the evolution of the wave function is unitary $\left|\psi\left(n \tau^{-}\right)\right\rangle=U(\tau) \mid \psi[(n-$ $\left.\left.1) \tau^{+}\right]\right\rangle$, where $U(\tau)=\exp (-i H \tau)$ (we set $\hbar=1$ in this paper). Let $\phi_{n}$ be the FDT amplitude, and this amplitude gives the probability $F_{n}=\left|\phi_{n}\right|^{2}$ of first detecting the particle in the $n$th attempt $[16,18]$. If the particle is detected with probability 1 (for further details, see Ref. [41]), which means $\sum_{n=1}^{\infty}\left|\phi_{n}\right|^{2}=1$, then the mean FDT time is $\langle t\rangle=$ $\tau \sum_{n=1}^{\infty} n\left|\phi_{n}\right|^{2}$. As we will soon recap, $\phi_{n}$ can be evaluated from a unitary evolution interrupted by projective measurements. Moreover, there exist a deep relation between $\phi_{n}$ and the unitary evolution without interrupting measurement [18].

The FDT amplitude $\phi_{t, n}$ for the evolution from $\left|\psi_{\text {in }}\right\rangle$ to $\left|\psi_{\mathrm{d}}\right\rangle$ and the FDR amplitude $\phi_{r, n}$ for the evolution from $\left|\psi_{\mathrm{d}}\right\rangle$ to $\left|\psi_{\mathrm{d}}\right\rangle$ read $[13,16,18]$

$$
\begin{aligned}
& \phi_{t, n}=\left\langle\psi_{\mathrm{d}}\left|\left(e^{-i \tau H} P\right)^{n-1} e^{-i \tau H}\right| \psi_{\mathrm{in}}\right\rangle, \\
& \phi_{r, n}=\left\langle\psi_{\mathrm{d}}\left|\left(e^{-i \tau H} P\right)^{n-1} e^{-i \tau H}\right| \psi_{\mathrm{d}}\right\rangle,
\end{aligned}
$$

with $P=1-D=1-\left|\psi_{\mathrm{d}}\right\rangle\left\langle\psi_{\mathrm{d}}\right| . P$ is the eraser operator it removes the part of the wave function on the detected state, corresponding to failed measurement. As the equations show, the unitary evolution in the detection free interval $\tau$ is interrupted by the operation $P$. The combined unitary evolution and the projection goes with the power $n-1$, corresponding to the $n-1$ prior failed measurements. Moreover, we define the unitary transition amplitude $v_{n}$ and the unitary return amplitude $u_{n}$ as

$$
\begin{aligned}
& v_{n}=\left\langle\psi_{\mathrm{d}}\left|e^{-\mathrm{in} H \tau}\right| \psi_{\mathrm{in}}\right\rangle, \\
& u_{n}=\left\langle\psi_{\mathrm{d}}\left|e^{-\mathrm{in} H \tau}\right| \psi_{\mathrm{d}}\right\rangle .
\end{aligned}
$$

These amplitudes describe transitions from the initial state to the detected state and from the detected state back to itself, free of any measurement. Using the $v_{n}$ and $u_{n}$, we expand
Eqs. (2) and (3) in $P$ which leads to an iteration equation known as the quantum renewal equation [18]:

$$
\begin{aligned}
& \phi_{t, n}=v_{n}-\sum_{j=1}^{n-1} \phi_{t, j} u_{n-j}, \\
& \phi_{r, n}=u_{n}-\sum_{j=1}^{n-1} \phi_{r, j} u_{n-j} .
\end{aligned}
$$

As in Eqs. (2) and (3) the subscript $r$ and $t$ denote the return and the transition probelms respectively. Note that the first terms $v_{n}, u_{n}$ on the right-hand side describe the unitary evolution between the initial state and the detected state and between the detected state to itself. The second terms describe all the former wave function returns to the detected state. These recursive equations, together with the exact function Eq. (38) for mean transition times, are used in the example section to find exact solutions of the problem. In order to solve the recursive equations a direct method is to transform the quantum renewal equation into the frequency (or $\omega$ ) space. Since the renewal equations consist of $\left\{v_{j}\right\}$ and $\left\{u_{j}\right\}$, we need to transform these quantities into $\omega$ space first. Using Eqs. (4) and (5) we have

$$
\begin{aligned}
& \tilde{v}(\omega):=\sum_{n=1}^{\infty} e^{i n \omega} v_{n}=\left\langle\psi_{\mathrm{d}}\left|\left(e^{i \tau H-i \omega}-1\right)^{-1}\right| \psi_{\mathrm{in}}\right\rangle, \\
& \tilde{u}(\omega):=\sum_{n=1}^{\infty} e^{i n \omega} u_{n}=\left\langle\psi_{\mathrm{d}}\left|\left(e^{i \tau H-i \omega}-1\right)^{-1}\right| \psi_{\mathrm{d}}\right\rangle .
\end{aligned}
$$

The analogous calculation for the amplitudes $\phi_{t, n}, \phi_{r, n}$ leads to

$$
\begin{aligned}
& \tilde{\phi}_{t}(\omega) \equiv \sum_{n=1}^{\infty} e^{i \omega n} \phi_{t, n}=\left\langle\psi_{\mathrm{d}}\left|A_{\omega}\right| \psi_{\mathrm{in}}\right\rangle, \\
& \tilde{\phi}_{r}(\omega) \equiv \sum_{n=1}^{\infty} e^{i \omega n} \phi_{r, n}=\left\langle\psi_{\mathrm{d}}\left|A_{\omega}\right| \psi_{\mathrm{d}}\right\rangle .
\end{aligned}
$$

where $A_{\omega}=\left(e^{i \tau H-i \omega}-P\right)^{-1}$. The initial state $\left|\psi_{\text {in }}\right\rangle$ distinguishes the return and transition problem. $A_{\omega}$ is related to the Green's function $\left(e^{i H \tau} / z-P\right)^{-1}$ of the nonunitary evolution [42]. Its poles are the solutions of $\operatorname{det}[\mathbb{1} / z-P U(\tau)]=0$. We will see later that these poles are essential for the evaluation of the mean FDT time. This property implies that the repeated measurement protocol can be related to open quantum systems, in the sense that the measurements acting on the system is equivalent to the interaction between environment and the system $[16,43,44]$. We believe that further research on this topic is worthwhile.

Using the identity $(1+B)^{-1}=1-B(1+B)^{-1}$, we obtain

$$
\begin{aligned}
\left\langle\psi_{\mathrm{d}}\left|A_{\omega}\right| \psi_{\mathrm{in}}\right\rangle & =\tilde{v}(\omega)-\tilde{u}(\omega)\left\langle\psi_{\mathrm{d}}\left|A_{\omega}\right| \psi_{\mathrm{in}}\right\rangle, \\
\left\langle\psi_{\mathrm{d}}\left|A_{\omega}\right| \psi_{\mathrm{d}}\right\rangle & =\tilde{u}(\omega)-\tilde{u}(\omega)\left\langle\psi_{\mathrm{d}}\left|A_{\omega}\right| \psi_{\mathrm{d}}\right\rangle .
\end{aligned}
$$

Then the generating functions for the amplitude $\phi_{t}$ and $\phi_{r}$ read

$$
\tilde{\phi}_{t}(\omega)=\frac{\tilde{v}(\omega)}{1+\tilde{u}(\omega)}, \quad \tilde{\phi}_{r}(\omega)=\frac{\tilde{u}(\omega)}{1+\tilde{u}(\omega)} .
$$

In the return problem, the initial state and detected state coincide, so the generating function only contains $\tilde{u}(\omega)$. Whereas 
in the transition problem the symmetry is broken, leading to the term $\tilde{v}(\omega)$ in the numerator.

The analytic continuation of the phase factor exp $(i \omega)$ from the unit disk to the parameter $z$ in the complex plane is convenient for further calculations. This leads to [18]

$$
\begin{aligned}
& \hat{\phi}_{t}(z)=\frac{\left\langle\psi_{\mathrm{d}}|\hat{U}(z)| \psi_{\mathrm{in}}\right\rangle}{1+\left\langle\psi_{\mathrm{d}}|\hat{U}(z)| \psi_{\mathrm{d}}\right\rangle}, \\
& \hat{\phi}_{r}(z)=\frac{\left\langle\psi_{\mathrm{d}}|\hat{U}(z)| \psi_{\mathrm{d}}\right\rangle}{1+\left\langle\psi_{\mathrm{d}}|\hat{U}(z)| \psi_{\mathrm{d}}\right\rangle},
\end{aligned}
$$

where $\hat{U}(z)=\sum_{n=1}^{\infty} z^{n} U(n \tau)=z U(\tau) /[\mathbb{1}-z U(\tau)]$ is the $Z$ (or discrete Laplace) transform of $U(n \tau)$. The difference between Eq. (15) and Eq. (16) is again only the numerator. Evaluating the generating function we may then use several approaches to find the statistical behavior of the process, see below [18].

\section{Pseudodegeneracy}

The degeneracy of the energy levels plays a crucial role in the problem [13]. For instance, a geometric symmetry of the graph can introduce such degeneracies. What is special here is that the measurement period $\tau$ leads to a new type of degeneracy of the distinct energy levels. This degeneracy is rooted in the stroboscopic sampling under investigation.

For an arbitrary Hamiltonian $H$ which has $w$ nondegenerate energy levels, the eigenvalues $\left\{E_{k}\right\}_{k=0, \ldots, w-1}$ of the Hamiltonian $H$ and the corresponding eigenstates $\left\{\left|E_{k l}\right\rangle\right\}_{k=0, \ldots, w-1}$ with $1 \leqslant l \leqslant g_{k}$, where $g_{k}$ is the degeneracy, can be used to express the matrix elements of Eq. (4) and Eq. (5) in spectral representation as

$$
\begin{gathered}
v_{n}=\sum_{k=0}^{w-1}\left\{\sum_{l=1}^{g_{k}}\left\langle\psi_{\mathrm{d}} \mid E_{k l}\right\rangle\left\langle E_{k l} \mid \psi_{\mathrm{in}}\right\rangle\right\} e^{-\mathrm{in} E_{k} \tau}, \\
u_{n}=\sum_{k=0}^{w-1}\left\{\sum_{l=1}^{g_{k}}\left|\left\langle\psi_{\mathrm{d}} \mid E_{k l}\right\rangle\right|^{2}\right\} e^{-\mathrm{in} E_{k} \tau} .
\end{gathered}
$$

These expressions are invariant under the change $E_{k} \tau \rightarrow$ $E_{k} \tau+2 \pi j$ for integer $j$. Thus, the eigenvalues $E_{k}, E_{k^{\prime}}$ are effectively degenerate if $E_{k} \tau=E_{k^{\prime}} \tau+2 \pi j$. Therefore, rather than the scaled eigenvalues $\left\{E_{k} \tau\right\}$ (which will be called simply eigenvalues subsequently), the back-folded eigenvalues $\left\{\bar{E}_{k} \tau\right\}$

$$
\bar{E}_{k} \tau=E_{k} \tau(\bmod \quad 2 \pi) \quad-\pi \leqslant \bar{E}_{k} \tau<\pi,
$$

determine the dynamics at fixed $\tau$. This can also be understood as the mapping $E_{k} \tau \rightarrow e^{-i E_{k} \tau}$ from the real axis to the unit circle on the complex plane [13] (see Fig. 2). Here it is possible to change the value of $\tau$ until $\tau=\tau_{c}$ which leads to $[13,18,41]$

$$
\left|E_{k}-E_{i}\right| \tau_{c}=2 \pi j,
$$

where $j$ is an integer. Thus, there are degeneracies of the backfolds eigenvalues for this critical $\tau_{c}$. Since the back-folded spectrum is relevant for the FDR or FDT and not the spectrum of $H$ itself, these degeneracies affect the discrete dynamics, even if the eigenvalues $\left\{E_{k}\right\}$ of $H$ are nondegenerate.

The quantum problem has a classical counterpart known as the first passage problem. The two problems exhibit vastly

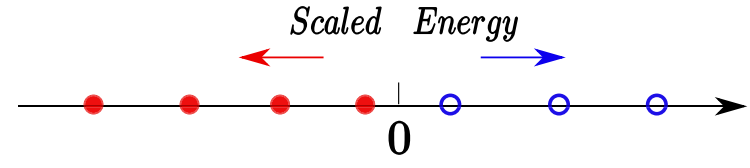

(a)
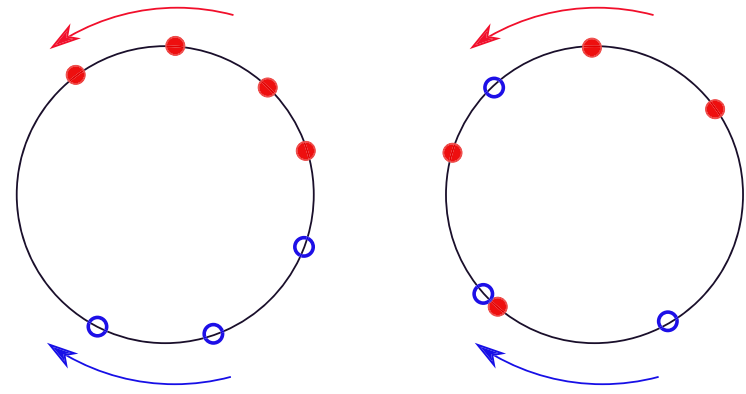

(b)

FIG. 2. Schematic behaviors of (a) the scaled Hamiltonian spectrum $E_{k} \tau$ and (b) the phase $e^{-i E_{k} \tau}$ under a change of the sampling time $\tau$. The arrows indicate the movements of the scaled energy levels $\left(E_{k} \tau\right)$ when increasing $\tau$. The positive (blue circles) and the negative (red dots) energy levels are well separated in (a). After mapping to the unit circle $E_{k} \tau \rightarrow e^{-i E_{k} \tau}$ they are not separated all the time, moving on the unit circle making fusion of the phases possible. In particular, the case (b) can lead to degeneracies in the back-folded spectrum and to very large mean transition times.

different behaviors, as might be expected. Let $P_{\text {det }}=$ $\sum_{n=1}^{\infty}\left|\phi_{n}\right|^{2}$ be the total detection probability. Unlike classical random walks on finite graphs, here one can find the total detection probability less than unity. The quantum particle will go to some "dark states", where they will never be detected $[41,42,45]$.

In Refs. [41,42] it was shown that $P_{\text {det }}<1$ when the Hilbert space is split into two subspaces dark and bright. The dark states can arise either from degeneracies of the energy spectrum or from energy levels that have no overlap with the detected state. The main focus of this paper is on cases where the total detection probability is unity (otherwise, the search is clearly not efficient). Thus in our system we have $P_{\text {det }}=1$, except for special sampling times, given by Eq. (20). On these sampling times the detection probability is suboptimal. Close to these sampling times the mean time for detection will diverge, and one of our goals is to understand this behavior.

\section{E. Zeros and poles}

From $\hat{\phi}(z)=\sum_{n=1}^{\infty} z^{n} \phi_{n}$ we extract the amplitude $\phi_{n}$ by the inverse transformation [18]

$$
\phi_{n}=\frac{1}{2 \pi i} \oint_{\gamma} \hat{\phi}(z) z^{-n-1} d z,
$$

where $\gamma$ is a counterclockwise closed contour around the circle of the complex plane with $|z|<1$, where $\hat{\phi}(z)$ is analytic. To perform the integration, we must analyze $\hat{\phi}(z)$. In Eqs. (15) and (16) the denominators only contain the state $\left|\psi_{\mathrm{d}}\right\rangle$ and not the initial condition $\left|\psi_{\text {in }}\right\rangle$, for both the FDR and FDT 
case. The poles outside the unit disk in turn will determine the relaxation pattern of $\phi_{n}$ (see below). To progress in our study of the transition problem we will use recent advances on the properties of the return problem $[13,37]$. For this purpose we study the connection between the return and the transition problem more explicitly. First, we define the overlap functions $p_{k}$ and $q_{k}$ of the initial and detected state as

$$
\begin{gathered}
q_{k}=\sum_{l=1}^{g_{k}}\left\langle\psi_{\mathrm{d}} \mid E_{k l}\right\rangle\left\langle E_{k l} \mid \psi_{\mathrm{in}}\right\rangle, \\
p_{k}=\sum_{l=1}^{g_{k}}\left|\left\langle\psi_{\mathrm{d}} \mid E_{k l}\right\rangle\right|^{2},
\end{gathered}
$$

which correspond to the distinct energy level $E_{k}$ with degeneracy $g_{k} \cdot q_{k}$ contains both detected and initial states while $p_{k}$ is only related to the detected state $\left|\psi_{\mathrm{d}}\right\rangle$. These expressions indicate that $p_{k}$ is real and nonnegative while $q_{k}$ is complex. The normalization of the energy eigenstates imply $\sum_{k=0}^{w-1} p_{k}=1$. On the other hand, $\sum_{k=0}^{w-1} q_{k}=0$, since the initial state and detected state are assumed to be orthogonal in the transition problem.

Next, we write the generating function in spectral representation as before, using eigenstates $\left|E_{k l}\right\rangle$ and the corresponding $g_{k}$-folded eigenvalues $E_{k}$. By multiplying both numerator and denominator $\prod_{k=0}^{w-1}\left(e^{i E_{k} \tau}-z\right)$, we express $\hat{\phi}_{t}(z)$ and $\hat{\phi}_{r}(z)$ as

$$
\hat{\phi}_{t}(z)=\frac{\mathcal{N}_{t}(z)}{\mathcal{D}(z)}, \quad \hat{\phi}_{r}(z)=\frac{\mathcal{N}_{r}(z)}{\mathcal{D}(z)} .
$$

Using $q_{k}$ and $p_{k}$ we can express $\mathcal{N}_{t}(z), \mathcal{N}_{r}(z)$, and $\mathcal{D}(z)$ as

$$
\begin{aligned}
& \mathcal{N}_{t}(z)=z \sum_{i=0}^{w-1} q_{i}\left[\prod_{k=0, k \neq i}^{w-1}\left(e^{i E_{k} \tau}-z\right)\right], \\
& \mathcal{N}_{r}(z)=z \sum_{i=0}^{w-1} p_{i}\left[\prod_{k=0, k \neq i}^{w-1}\left(e^{i E_{k} \tau}-z\right)\right], \\
& \mathcal{D}(z)=\sum_{i=0}^{w-1} p_{i} e^{i E_{i} \tau}\left[\prod_{k=0, k \neq i}^{w-1}\left(e^{i E_{k} \tau}-z\right)\right] .
\end{aligned}
$$

The only difference between the $\mathcal{N}_{r}(z)$ and $\mathcal{N}_{t}(z)$ is that the $q_{i}$ in the former is replaced with $p_{i}$ in the latter. So $p_{i}$ and $q_{i}$ characterize the generating function of the return and the transition problem. $\mathcal{N}_{r}(z)$ and $\mathcal{D}(z)$ share the same multiplication term, each depending on the same group of real numbers $p_{i}$ and $\left\{E_{i}\right\}$. A straightforward calculation shows that the two polynomials are related [18]:

$$
\mathcal{D}(z)=(-1)^{w-1} e^{i \sum_{j} E_{j} \tau} z^{w}\left[\mathcal{N}_{r}\left(1 / z^{*}\right)\right]^{*} .
$$

From Eqs. (15) and (16) the poles of the return and transition problem are identical. These poles, denoted by $Z_{i}$, are found from the solutions of $\mathcal{D}(Z)=0$. We also define the zeros of the generating function in the return problem, denoted by $z_{r, i}$. The latter are given by $\mathcal{N}_{r}(z)=0$. From Eq. (28), $\mathcal{D}(z)=(-1)^{w-1} e^{i \sum_{j} E_{j} \tau} z^{w}\left[\mathcal{N}_{r}\left(1 / z^{*}\right)\right]^{*}=0$ yields $Z_{i}=1 / z_{r, i}^{*}$. Hence transition poles $Z_{i}$ are given by

$$
Z_{i}=\frac{1}{z_{r, i}^{*}}, \quad z_{r, i} \neq 0
$$

The key point is that the $\left\{Z_{i}\right\}$ describe both the transition problem investigated here and the return problem [13]. Subsequently, we write $z_{r, i}$ as $z_{i}$ for simplicity. Equation (29) gives us a way to find the poles $Z_{i}$ which are essential for the amplitude $\phi_{n}$, namely using the return zeros $z_{i}$, which have been studied already in the return problem [13,37].

\section{F. Charge theory}

As already discussed before, the central goal is to determine the zeros $\left\{z_{i}\right\}$. A very helpful method in this regard was proposed by Grübaum et al. [13], who mapped the return problem to a classical charge theory. More importantly, the classical charge theory provides an intuitive physical picture from which we can understand the behavior of the poles. Using Eq. (26) for the zeros of $\mathcal{N}_{r}(z)$ with some rearrangement, we have $z \sum_{k=0}^{w-1} p_{k} /\left(e^{i E_{k} \tau}-z\right)=0$. Neglecting the trivial zero at the origin we must solve

$$
\mathcal{F}(z)=\sum_{k=0}^{w-1} \frac{p_{k}}{e^{i E_{k} \tau}-z}=0 .
$$

$\mathcal{F}(z)$ can be considered as a force field with unit test charge in the complex plane, stemming from charges $p_{k}$ whose locations are $e^{i E_{k} \tau}$ on the unit circle. Then the zeros $\left\{z_{i}\right\}$ of $\mathcal{N}_{r}(z)$ are the stationary points of this force field. Since there are $w$ charges which corresponds to the number of the discrete energy levels, we get $w-1$ stationary points in this force field from Eq. (30). All the zeros are inside the unit disk, which is rather obvious since all the charges have the same sign $\left(p_{k}>0\right)$. The physical significance of this is that the modes of the problem decay. More precisely, the zeros are within a convex hull, whose edge is given by the position of the charges, hence $\left|z_{i}\right|<1$. Then Eq. (29) implies $\left|Z_{i}\right|>1$, i.e., the poles lie outside the unit circle.

\section{FDT TIME}

In this section we focus on finding the general expression for the mean FDT time. As mentioned, we assume $\left\langle\psi_{\mathrm{d}} \mid \psi_{\mathrm{in}}\right\rangle=$ 0 which is the definition of "transition". Since $\langle t\rangle=\tau\langle n\rangle=$ $\tau \sum_{n=1}^{\infty} n\left|\phi_{n}\right|^{2}$, the first step is to find the amplitudes $\phi_{n}$, describing the detection probability for the $n$th attempt. We start from the generating function of the FDT problem Eq. (15) with Eqs. (24), (25), (26), and (27):

$$
\hat{\phi}_{t}(z)=\frac{z \sum_{i=0}^{w-1} q_{i}\left[\prod_{k=0, k \neq i}^{w-1}\left(e^{i E_{k} \tau}-z\right)\right]}{\sum_{i=0}^{w-1} p_{i} e^{i E_{i} \tau}\left[\prod_{k=0, k \neq i}^{w-1}\left(e^{i E_{k} \tau}-z\right)\right]} .
$$

We now define the polynomial $\mathcal{G}(z)$ related to the numerator $\mathcal{N}_{t}(z)$ in Eq. (25) according to

$$
\mathcal{N}_{t}(z)=z \sum_{i=0}^{w-1} q_{i}\left[\prod_{k=0, k \neq i}^{w-1}\left(e^{i E_{k} \tau}-z\right)\right]=z \mathcal{G}(z) .
$$

Using $\sum_{i} q_{i}=0$, it is not difficult to show that $\operatorname{deg}[\mathcal{D}(z)]>$ $\operatorname{deg}[\mathcal{G}(z)]$ (see details in Appendix A). We rewrite the generating function by "general partial decomposition" for isolated poles of the denominator and a polynomial $\mathcal{G}(z)$ of order smaller than $w-1$. Using the $w-1$ poles $\left\{Z_{i}\right\}$ we found before, we rewrite $\mathcal{D}(z)=\beta\left(z-Z_{1}\right)\left(z-Z_{2}\right) \cdots(z-$ 
$\left.Z_{w-1}\right)\left(\beta\right.$ is the coefficient of $z^{w-1}$, see Appendix A). Then we obtain

$$
\frac{\mathcal{G}(z)}{\beta\left(z-Z_{1}\right) \cdots\left(z-Z_{w-1}\right)}=\sum_{i=1}^{w-1} \frac{C_{i}}{Z_{i}\left(z-Z_{i}\right)},
$$

where $C_{i}$ is given by

$$
\begin{aligned}
C_{i} & =\frac{Z_{i}}{2 \pi i} \oint_{\gamma_{i}} \frac{\mathcal{G}(z)}{\beta\left(z-Z_{1}\right) \cdots\left(z-Z_{w-1}\right)} d z \\
& =\frac{\mathcal{N}_{t}\left(Z_{i}\right)}{\beta} \prod_{k \neq i} \frac{1}{Z_{i}-Z_{k}} .
\end{aligned}
$$

The contours $\gamma_{i}$ enclose only $Z_{i}$ but not $\left\{Z_{k}\right\}_{k \neq i}$. Since $Z_{i}$ is the pole of $[\mathcal{D}(z)]^{-1}$, we can rewrite the multiplication as $\beta^{-1} \prod_{k \neq i}\left(Z_{i}-Z_{k}\right)^{-1}=\left.\left[\partial_{z} \mathcal{D}(z)\right]^{-1}\right|_{z=Z_{i}}$, hence

$$
C_{i}=\frac{\mathcal{N}_{t}\left(Z_{i}\right)}{\left.\partial_{z} \mathcal{D}(z)\right|_{z=Z_{i}}} .
$$

This allows us to rewrite the generating function as $\hat{\phi}_{t}(z)=$ $\sum_{i=1}^{w-1} z C_{i} /\left[Z_{i}\left(z-Z_{i}\right)\right]$, where $\hat{\phi}_{t}(z)$ is decomposed into the summation of the $z C_{i} /\left[\left(z-Z_{i}\right) Z_{i}\right]$ in which there is only one pole in the denominator. With Eq. (21) the first detection amplitude reads

$$
\phi_{n}=\sum_{i=1}^{w-1} \frac{C_{i}}{2 \pi i} \oint_{\gamma} \frac{z^{-n}}{Z_{i}\left(z-Z_{i}\right)} d z=-\sum_{i=1}^{w-1} C_{i} Z_{i}^{-n-1} .
$$

In a formal way this is the solution of the first detection transition problem for any finite dimensional Hilbert space, though below we will focus on the mean transition time only. The probability of finding the quantum state $\left|\psi_{\mathrm{d}}\right\rangle$ at the $n$th attempt is $F_{n}=\left|\phi_{n}\right|^{2}$. Summing the geometric series the total detection probability $P_{\text {det }}=\sum_{n=1}^{\infty} F_{n}$ is

$$
P_{\mathrm{det}}=\sum_{i, j=1}^{w-1} \frac{C_{i} C_{j}^{*}}{\left(Z_{i} Z_{j}^{*}-1\right) Z_{i} Z_{j}^{*}} .
$$

As mentioned, other methods for finding $P_{\text {det }}$ were considered in Ref. [41]. For a finite system, it was shown that $P_{\text {det }}$ is independent of the measurement interval $\tau$ except for the special resonant points in Eq. (20) where new degeneracy appears. In finite-dimensional Hilbert space, the total detection probability is $P_{\text {det }}=1$ when all the energy levels have projection on the detected state and the back-folded spectrum is not degenerate.

In this paper we treat the case when the total detection probability is 1 , and this means that the detection of the quantum state in an experiment is guaranteed. We can define the mean FDT time $\langle t\rangle=\langle n\rangle \tau$, where $\langle n\rangle$ is the mean of the number of detection attempts. For convenience, we call $\langle n\rangle$ the mean of FDT time in the rest of the paper due to the simple relation between the $\langle t\rangle$ and $\langle n\rangle$. From $\langle n\rangle=$ $\sum_{n=1}^{\infty} n\left|\phi_{n}\right|^{2}$, together with Eq. (36), we find

$$
\langle n\rangle=\sum_{i, j=1}^{w-1} \frac{C_{i} C_{j}^{*}}{\left(Z_{i} Z_{j}^{*}-1\right)^{2}} .
$$

Equations (30), (35), and (38) expose how the mean FDT time depends on the spectrum of $H$, the initial state $\left|\psi_{\text {in }}\right\rangle$, the detected state $\left|\psi_{\mathrm{d}}\right\rangle$, and the sampling time $\tau$. Since in general the denominator of Eq. (38) is vanishing when some $Z_{k}$ is approaching the unit circle, we may have some critical scenarios, where the $\langle n\rangle$ can be asymptotically computed by neglecting nondiverging terms in the formal formula Eq. (38). This leads to simpler formulas but with more physical insights. We will investigate these cases in the following sections.

\section{RELATION OF THE MEAN FDT TIME AND THE FDR VARIANCE}

There is a general relation between the mean FDT time $\langle n\rangle$ and the fluctuations of the return time, and we investigate this relation showing that it is particularly useful when both are large. First, we reformulate some of the main equations which we will use later. The variance of the FDR time is [13]

$$
V_{r}=\left\langle n^{2}\right\rangle_{r}-\langle n\rangle_{r}^{2}=\sum_{i, j=1}^{w-1} V_{i, j},
$$

where $V_{i, j}=2 /\left(Z_{i} Z_{j}^{*}-1\right)$. Using Eq. (37), $P_{\text {det }}$ can be written in terms of summations over matrix elements of $P_{i, j}$ :

$$
P_{\mathrm{det}}=\sum_{i, j} P_{i, j}, \quad P_{i, j}=\frac{C_{i} C_{j}^{*}}{\left(Z_{i} Z_{j}^{*}-1\right) Z_{i} Z_{j}^{*}} .
$$

As mentioned, we now assume that there are no dark states in the system, such that the total detection probability $P_{\text {det }}$ is unity, see conditions in Refs. [41,42]. This implies the absence of degeneracy in the spectrum. Using Eq. (38), the matrices $P_{i, j}$ and $V_{i, j}$ also give the mean FDT time:

$$
\langle n\rangle=\frac{1}{2} \sum_{i, j=1}^{w-1} Z_{i} Z_{j}^{*} P_{i, j} V_{i, j} .
$$

This equation relates the $\langle n\rangle$ and the matrix $V_{i, j}$, and the latter also gives the variance of the return in Eq. (39). This indicates that the fluctuations of the FDR time reveal the characteristics of the mean FDT time.

Below we show cases where one element of the summation is dominating $V_{r} \sim V_{s, s}$ and $\left|Z_{s}\right| \rightarrow 1$ (the subscript $s$ stands for single), such that

$$
\langle n\rangle \sim \frac{P_{s, s}}{2} V_{s, s} \sim \frac{P_{s, s}}{2} V_{r} .
$$

This is similar to the Einstein relation in the sense that diffusivity (a measure of fluctuations) is related to mobility (a measure of the average response). This effect is found when a single zero of the electrostatic field is approaching the unit circle, and in this case this zero is dominating both the return problem and the transition problem. In Sec. VII we find exact expressions for $\langle n\rangle$ and $V_{r}$ satisfying Eq. (42), based on several physical scenarios.

After obtaining the general results Eqs. (38) and (41), we will focus on the diverging mean FDT time, where the asymptotic $\langle n\rangle$ and its relation to $V_{r}$ is found, i.e., Eq. (42). Equation (38) implies a divergent mean FDT time when $\left|Z_{s}\right| \rightarrow 1$, as mentioned since $\left|Z_{s}\right|=1 /\left|z_{s}\right|$, where $z_{s}$ is the stationary point of the electrostatics field, the question is under what condition there is a stationary point close to the unit circle. Next we will investigate three scenarios where $\left|Z_{S}\right| \rightarrow 1$, 
using the electrostatic picture. We distinguish them into the following cases: (1) a weak charge scenario, (2) two charges merging picture, and, finally, (3) one big charge theory.

\section{WEAK CHARGE}

In electrostatics, when one charge becomes much smaller than all other charges, one of the stationary points will be close to the weak charge [13]. This is presented in Fig. 3, where the blue charge indicates the weak charge. Note that in the figure we present the zero inside the unit disk, hence its corresponding pole is $Z_{0}=1 / z_{0}^{*}$. In analogy, the stationary point of the Moon-Earth system is much closer to the Moon than to the Earth. We denote this charge $p_{0}$ and the stationary point $z_{0}$. The corresponding energy level of this weak charge is $E_{0}$ and its location is $\exp \left(i E_{0} \tau\right)$ on the unit circle. Since $z_{0} \rightarrow e^{i E_{0} \tau}$, from Eq. (29) the reciprocal pole $\left|Z_{0}\right|=1 /\left|z_{0}\right| \rightarrow$ 1. Using Eq. (38), the asymptotic mean of the mean FDT time is

$$
\langle n\rangle \sim \frac{\left|C_{0}\right|^{2}}{\left(\left|Z_{0}\right|^{2}-1\right)^{2}},
$$

when $p_{0} \rightarrow 0$ and $\left|q_{0}\right| / p_{0} \gg 1$. Here we assume $\left|C_{0}\right|^{2} /\left(\left|Z_{0}\right|^{2}-1\right)$ is the dominating part of $\langle n\rangle$, and all other terms in Eq. (38) are negligible. To find the exact expression of $\langle n\rangle$, we first need to find the pole $Z_{0}$. Using Eq. (30) together with perturbation theory presented in Appendix B, we get

$$
Z_{0} \sim e^{i \tau E_{0}}+\frac{p_{0} e^{2 i \tau E_{0}}}{\sum_{k=1}^{w-1} p_{k} /\left(e^{-i \tau E_{0}}-e^{-i \tau E_{k}}\right)},
$$

Since $p_{0} \ll 1, e^{i E_{0} \tau}$ is the leading part of $Z_{0}$. Hence the pole $Z_{0}$ is located very close to the weak charge as we expect from basic electrostatics. The other $w-1$ charges give a small disturbance to $Z_{0}$ if they are not close to the weak charge. Substituting $Z_{0}$ into Eq. (35), the coefficient $C_{0}$ can be obtained (see Appendix B). Then using the normalization condition $\sum_{k} p_{k}=1$ and $1 /(1-\exp [i x])=1 / 2+i \cot [x / 2] / 2$, we get from Eq. (43) the mean FDT time

$$
\langle n\rangle \sim \frac{\left|q_{0}\right|^{2}}{4 p_{0}^{2}}\left(1+\left\{\sum_{k=1}^{w-1} p_{k} \cot \left[\left(E_{k}-E_{0}\right) \tau / 2\right]\right\}^{2}\right) .
$$

The prefactor $\left|q_{0}\right|^{2} / 4 p_{0}^{2}$ depends on $q_{0}$ and $p_{0}$ defined in Eqs. (22) and (23), and they rely only on the stationary states of energy level $E_{0}$, the initial and final states, but not on the other energy states of the system. This prefactor is the envelope of the mean FDT time as the $\cot ()$ solution is oscillating when we modify $\tau$. From our assumption $\left|q_{0}\right| / p_{0} \gg 1$ the value of this envelope is large. The summation in the bracket shows that $\langle n\rangle$ depends on all charges as expected.

As mentioned when Eq. (20) holds we get the merging of two phases on the unit circle a case we will study in detail in the next section. In the vicinity of this point the mean FDT time diverges. So what is the physics for this divergence? We have shown before when two energy levels coalesce, the total detection probability $P_{\text {det }}$ is not unity, which means the quantum particle goes to "dark states" in the Hilbert space [41]. This divergence reflects that the total detection probability $P_{\text {det }}$ deviates from 1 , indicating that one or more states are not accessible by the quantum walker. We will see this connection in some examples below.

\section{TWO MERGING CHARGES}

Another case with a pole close to the unit circle is when the phases of two charges, denoted by $p_{a}$ and $p_{b}$, satisfy the resonance condition $\exp \left(i E_{a} \tau\right) \simeq \exp \left(i E_{b} \tau\right)$. Here $E_{a}$ and $E_{b}$ are a pair of energy levels in the system that satisfy the merging condition. Note again that in Fig. 3 we present the zero $z_{p}$ related to the pole according to $Z_{p}=1 / z_{p}^{*}$. As mentioned, this means that we are close to a degeneracy of the back-folded spectrum. It can be achieved by modifying $H$ or the sampling time $\tau$. Then the small parameter $\delta=$
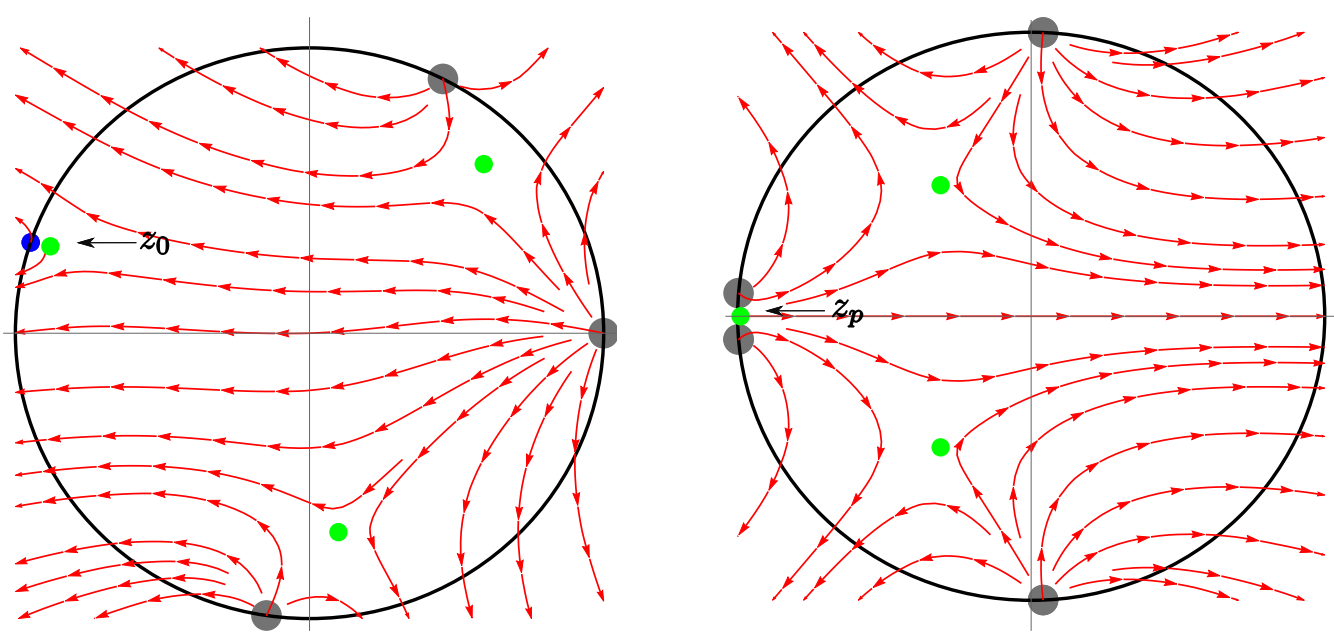

FIG. 3. Schematic plot of the cases where the zeros are close to the unit disk. The red curves are the electric fields lines, created by the charges on the unit circle. And this field vanishes at the green points which are the stationary points defined in Eq. (30). From electrostatics if a charge (blue) is weak, one stationary point denoted $z_{0}$ will be close to this weak charge (left). Another case presented on the right is when two charges are merging. Also here we have one zero $z_{p}$ close to the unit circle (right). The two merging charges on the right are on $\exp \left(i E_{a} \tau\right) \simeq \exp \left(i E_{b} \tau\right)$, where $E_{a}$ and $E_{b}$ are two energy levels in the system. 
$\left(\bar{E}_{b}-\bar{E}_{a}\right) \tau / 2$ measures the angular distance between the two phases. When the two charges merge, a related pole denoted $Z_{p}$ (subscript $p$ is for pair of merging charges), will approach the unit circle $\left|Z_{p}\right| \rightarrow 1$. Using Eq. (38), for the mean FDT time $\langle n\rangle$, we get

$$
\langle n\rangle \sim \frac{\left|C_{p}\right|^{2}}{\left(\left|Z_{p}\right|^{2}-1\right)^{2}}, \quad \delta \rightarrow 0 .
$$

Here we use the assumption that a single pole is approaching the unit disk, and the others can be neglected. To find the pole $Z_{p}$, we first treat the charge field as a two-body system. Because by our assumption all other charges are far away from the two merging charges. Then we take the background charges into consideration. Using the two-body hypothesis together with Eq. (29), we find in perturbation theory $Z_{p} \sim$ $Z_{p}^{(0)}+Z_{p}^{(1)}$ (see Appendix C). Here $Z_{p}^{(0)}$ and $Z_{p}^{(1)}$ are defined in Appendix $C$ in Eq. (C7). Plugging $Z_{p}$ into Eq. (35) yields for the coefficient $C_{p} \sim 2 \delta\left|q_{a} p_{b}-q_{b} p_{a}\right| /\left(p_{a}+p_{b}\right)^{2}$, where $\left|C_{p}\right|$ is determined by the phase difference, charges and $q_{k}$. Since $\delta$ is a small parameter, $\left|C_{p}\right|$ also becomes small when two charges merge. Substituting $C_{p}$ and $Z_{p}$ into Eq. (46), the mean FDT time becomes

$$
\langle n\rangle \sim \frac{\left(p_{a}+p_{b}\right)^{2}\left|q_{a} p_{b}-q_{b} p_{a}\right|^{2}}{p_{a}^{2} p_{b}^{2}} \frac{1}{\tau^{2}\left(\bar{E}_{b}-\bar{E}_{a}\right)^{2}} .
$$

It should be noted that this formula does not include the background, which is quite different from the weak charge case. When two charges are merging, the expected transition time $\langle n\rangle$ diverges since $\left(\bar{E}_{b}-\bar{E}_{a}\right)^{2} \tau^{2}$ is small. The term $\left|q_{a} p_{b}-q_{b} p_{a}\right|^{2}$ comes from interference. At the special case $\left|q_{a} p_{b}-q_{b} p_{a}\right|^{2}=0$ we have an elimination of the resonance, meaning that the effect of divergence of $\langle n\rangle$ might be suppresses.

\section{RELATION BETWEEN MEAN FDT TIME AND FDR FLUCTUATIONS}

When there is only one pole dominating, simple relations between the mean FDT time and the fluctuations of the FDR time are found. We start from the general relation Eq. (41). When the pole $\left|Z_{s}\right| \rightarrow 1$ we have Eq. (42). Here $Z_{s}$ is a single pole approaching the unit circle, as mentioned, it could be either $Z_{p}$ for two merging charges or $Z_{0}$ for one weak charge. $P_{s, s}$ in Eq. (37) is the diagonal term of the matrix $\left\{P_{i, j}\right\}$, which is real and positive. Based on Secs. V and VI we can get exact expressions for $P_{s, s}$ under different circumstances.

In the weak charge regime, $P_{s, s} \sim\left|C_{0}\right|^{2} /\left(\left|Z_{0}\right|^{2}-1\right)$. Substituting the $C_{0}$ and $Z_{0}$ into $P_{s, s}$, the ratio of the mean FDT time and the FDR variance reads

$$
\frac{\langle n\rangle}{V_{r}} \sim \frac{\left|q_{0}\right|^{2}}{2 p_{0}} .
$$

When the energy level $E_{0}$ is not degenerate, we have

$$
\frac{\langle n\rangle}{V_{r}} \sim \frac{\left|\left\langle\psi_{\text {in }} \mid E_{0}\right\rangle\right|^{2}}{2} .
$$

From Eq. (48) and Eq. (45), we can get the expression of $V_{r}$, which confirms the result for $V_{r}$ in Ref. [37]. The beauty of Eq. (49) is that it only depends on the overlap of the initial state $\left|\psi_{\text {in }}\right\rangle$ and $\left|E_{0}\right\rangle$. So how we prepare the quantum particle is of great importance for the mean FDT time. In other words, the quantum particle will remember its history, unlike the classical walker. Furthermore, $\left|\left\langle\psi_{\text {in }} \mid E_{0}\right\rangle\right|^{2} / 2<1 / 2$ which implies that the mean FDT time is bounded by one half of the FDR variance.

For the two merging charges scenario we have $P_{s, s} \sim$ $\left|C_{p}\right|^{2} /\left(\left|Z_{p}\right|^{2}-1\right)$. Substituting the $C_{p}$ and $Z_{p}$ into $P_{s, s}$, we have

$$
\frac{\langle n\rangle}{V_{r}} \sim \frac{\left|q_{a} p_{b}-q_{b} p_{a}\right|^{2}}{2\left(p_{a}+p_{b}\right) p_{a} p_{b}} .
$$

From Eqs. (47) and (50) we get an expression for $V_{r}$, which was also derived in Ref. [37]. Here the initial state $\left|\psi_{\text {in }}\right\rangle$ plays an important role because $q_{a}$ and $q_{b}$ are related to the initial state (unlike $p_{a}$ and $p_{b}$ ). Under some special symmetry of the system we can get $p_{a} / q_{a}=p_{b} / q_{b}$, such that $\left|q_{a} p_{b}-q_{b} p_{a}\right|^{2}=$ 0 . As mentioned, this reflects a elimination of the resonance because $\langle n\rangle$ will tend to some small values, while the FDR variance diverges.

Remark. We may start from Eqs. (38) and (39), if one of the poles is denoted $Z_{s}$ and is close to the unit circle. Then we have roughly $\langle n\rangle \sim\left|C_{s}\right|^{2} /\left(\left|Z_{s}\right|^{2}-1\right)^{2}$ and $V_{r} \sim 2 /\left(\left|Z_{s}\right|^{2}-1\right)$. The relation of the mean FDT time and the FDR variance is $\langle n\rangle \sim\left|C_{s}\right|^{2} V_{r}^{2} / 4$, i.e., $\langle n\rangle$ is proportional to $V_{r}^{2}$. This intuition does not reveal the real physics, since for a divergent $V_{r}$ we get $\left|C_{s}\right| \rightarrow 0$.

\section{EXAMPLES}

As an application of our general theory we consider tightbinding models on simple graphs.

\section{A. Two-level system}

The first example is a quantum walk on a two-site graph [see Fig. 4(a)] (i.e., a two-level system). The Hamiltonian of this system reads

$$
H=-\gamma(|0\rangle\langle 1|+| 1\rangle\langle 0|+U| 0\rangle\langle 0|) .
$$

It describes a quantum particle hopping between two sites 0 and 1 , where a potential $U$ is added at site 0 . This model also presents a spin $1 / 2$ in a field.

We prepare the initial quantum state as $|0\rangle$, which means that the particle is on site 0 . The detector is set to detect the particle at site 1 , i.e., the detected state is $|1\rangle$. From Eq. (51) the energy spectrum of the system is (we set $\gamma=1$ subsequently): $E_{0}=\left(-U-\sqrt{U^{2}+4}\right) / 2$ and $E_{1}=(-U+$ $\left.\sqrt{U^{2}+4}\right) / 2$. In the large $U$ limit, where $E_{0} \rightarrow-U$ and $E_{1} \rightarrow 0$, the two energy levels $E_{0}$ and $E_{1}$ are separated. From Eq. (23) the charge $p_{0}=1 /\left(E_{0}^{2}+1\right)$ and from normalization $p_{1}=1-p_{0}$. When we increase the value of the potential $U$, the charge $p_{0} \rightarrow 0$, which represents a weak charge in the system. From Eq. (22) we have $q_{0}=E_{0} /\left(E_{0}^{2}+\right.$ 1) and $q_{1}=E_{1} /\left(E_{1}^{2}+1\right)$. The ratio $\left|q_{0}\right| / p_{0}$ is $\left|E_{0}\right|$,which is our dimensionless variable growing with the potential $U$. From Eq. (45) the mean FDT time of this simple two-level system is

$$
\langle n\rangle \sim \frac{U^{2}}{4}\left[1+\cot ^{2}(U \tau / 2)\right] .
$$




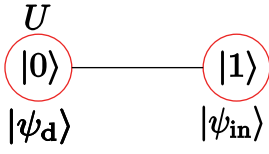

(a)

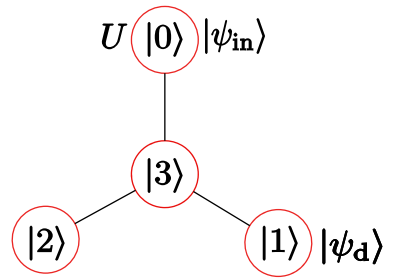

(b)

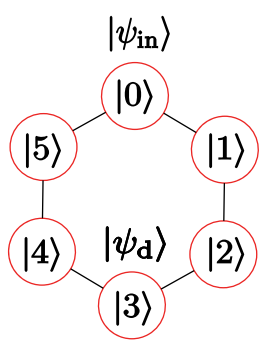

(c)

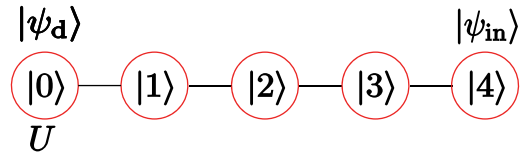

(d)
FIG. 4. Schematic models. We perform the calculations on different graphs, which represent tight-binding models defined in the text. The quantum particle is prepared in the initial state $\left|\psi_{\text {in }}\right\rangle$ and we set the detector to detect the state $\left|\psi_{\mathrm{d}}\right\rangle . U$ is the strength of potential well or potential barrier we set in the system. (a) Two-level model. (b) Y-shaped molecule. (c) Benzenelike ring. (d) Linear five-site molecule.

$\langle n\rangle$ becomes larger as we increase $U$, indicating the potential well blocks the propagation of the wave function, making it hard to find the particle at the detected state. In Eq. (52), when $U \tau$ is close to $2 \pi k, k=1,2, \ldots$, the mean FDT time diverges. Note that $U \tau=2 \pi k$ is the condition for exceptional points [Eq. (20)], in the limit of large $U$. At these exceptional points, the total detection probability $P_{\text {det }}$ drops from 1 to 0 . Using Eqs. (30) and (38), we can find the exact expression of the mean FDT time: $\langle n\rangle=\left\{q_{0}^{2}+q_{1}^{2}+2 q_{0} q_{1} \cos \left[\left(E_{0}-\right.\right.\right.$ $\left.\left.\left.E_{1}\right) \tau\right]\right\} /\left\{4 p_{0}^{2} p_{1}^{2}\left[1-\cos \left(E_{0} \tau-E_{1} \tau\right)\right]^{2}\right\}$.

Choosing the sampling frequency $1 / \tau=1 / 3$, the exact $\langle n\rangle$ can be obtained either from the quantum renewal equation Eq. (6) or our first main result Eq. (38). Here we use the latter formula, and the result is visualized in Fig. 5 (left $y$ axis). In the vicinity of the exceptional points the total detection probability drops from the unity and the mean FDT time diverges.

\section{B. Y-shaped molecule}

The next example is the $\mathrm{Y}$-shaped molecule, where the quantum particle can jump from states $|0\rangle,|1\rangle,|2\rangle$ to state $|3\rangle$ and vice versa [see Fig. 4(b)]. We add a potential $U$ at site 0 . Then the Hamiltonian of the $\mathrm{Y}$-shaped molecule reads

$$
H=-\gamma\left(U|0\rangle\left\langle 0\left|+\sum_{i=1}^{3}\right| 3\right\rangle\left\langle i\left|+\sum_{j=1}^{3}\right| j\right\rangle\langle 3|\right) .
$$

We prepare the quantum particle in the state $\left|\psi_{\text {in }}\right\rangle=|0\rangle$ and the detection is performed in the state $|1\rangle$. Due to the mirror symmetry of $\mathrm{Y}$-shaped molecule, the energy level $E_{3}=0$. Other energy levels $E_{0}, E_{1}$, and $E_{2}$ are given by the roots of

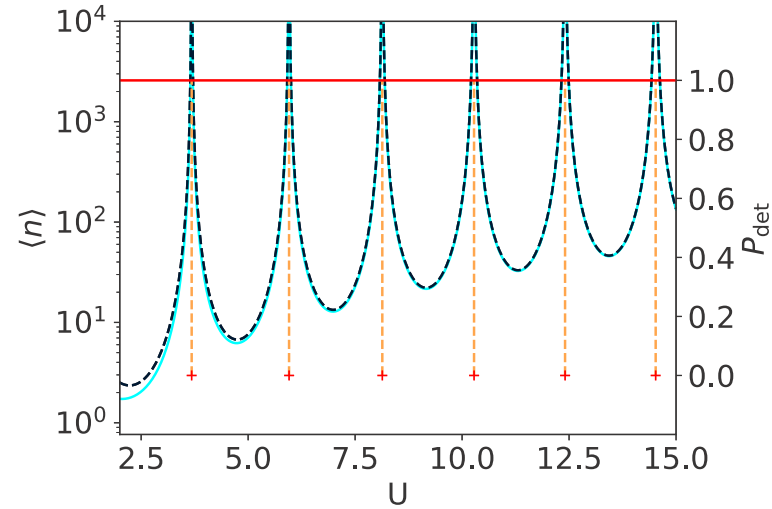

FIG. 5. The mean FDT time $\langle n\rangle$ (left $y$ axis) and total detection probability $P_{\text {det }}$ (right $y$ axis) versus the potential $U$ of the two-level system for the transition from $|1\rangle \rightarrow|0\rangle$ in Fig. 4(a). Here we fix $\tau=3$. The exact mean FDT time (black dashed line) meets quite well with our theoretical result Eq. (52) (cyan solid line) when $\langle n\rangle$ is large, corresponding to large $U$ ( $U>2.5$ in the figure). Close to the exceptional points $U \sim 2 \pi k / \tau, k=1,2, \ldots$, where the back-fold energy levels are degenerate, the total detection probability (red solid line) drops to $P_{\text {det }}=0$ and $\langle n\rangle$ diverges as we expected.

the equation $E^{3}+U E^{2}-3 E-2 U=0$. When $U$ is large, we have $E_{0} \sim-U, E_{1} \sim \sqrt{2}$, and $E_{2} \sim-\sqrt{2}$. From Eq. (23) the charges are $p_{0} \rightarrow 0, p_{1} \rightarrow 1 / 4, p_{2} \rightarrow 1 / 4$, and $p_{3} \rightarrow 1 / 2$. The appearance of the weak charge $p_{0}$ is because one of the eigenstate is nearly localized on $|0\rangle$, more specifically $\left|E_{0}\right\rangle \simeq|0\rangle$. The exact numerical values of both energy levels $\left\{E_{i}\right\}$ and charges $\left\{p_{i}\right\}$ are shown in Appendix A in Fig. 10. Using Eq. (45) the mean FDT time of the $Y$-shaped molecule reads

$$
\langle n\rangle \sim \frac{\left|q_{0}\right|^{2}}{4 p_{0}^{2}}\left(1+\left\{\sum_{i=1}^{3} p_{i} \cot \left[\left(E_{i}-E_{0}\right) \tau / 2\right]\right\}^{2}\right) .
$$

The initial site and detected site are not symmetric because of the potential $U$. This implies $\left|\left\langle E_{0} \mid 0\right\rangle\right| \gg\left|\left\langle E_{0} \mid 1\right\rangle\right|$ and $\left|q_{0}\right| / p_{0} \gg 1$. When two energy levels are coalescing Eq. (54) diverges. The prefactor in Eq. (54) indicates the asymptotic tendency of the mean FDT time versus the potential $U$ (see Fig. 6), which should be observed experimentally. We denote this prefactor as the weak charge envelope $\langle n\rangle_{e}$. From Eq. (48) the relation between the mean FDT time and the FDR variance gives

$$
\langle n\rangle / V_{r} \sim 1 / 2 .
$$

To plot the solution of this example, we solve the quantum renewal equations exactly, as was done in Sec. VIII A, here we choose the sampling period $\tau=3$. The value of potential well $U$ goes from 2 to 12. As shown in Fig. 6, Eqs. (54) and (55) work well in the weak charge regime where $U$ is large.

\section{Benzene-type ring}

For the third model we consider the Benzene-type ring which has six spacial states $|0\rangle,|1\rangle, \ldots,|5\rangle$ [see Fig. 4(c)]. We use periodic boundary conditions and thus from the site labeled $x=5$ the particle may hop either to the origin $x=0$ 

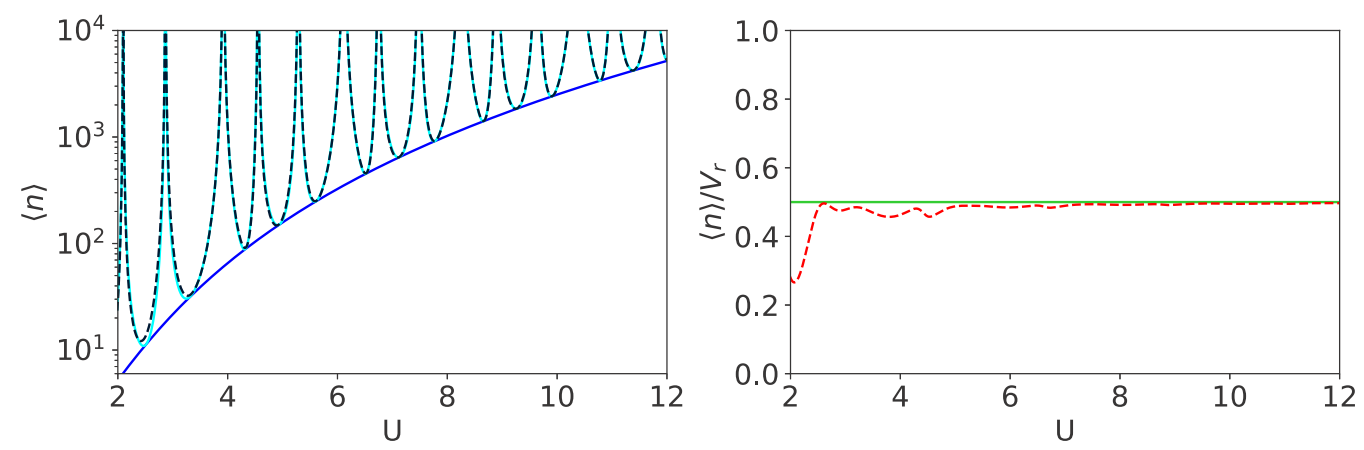

FIG. 6. The mean FDT time $\langle n\rangle$ (left) and the ratio of the mean FDT time and the FDR variance $\langle n\rangle / V_{r}$ (right) versus $U$ for the Y-shaped model. Here we choose $\tau=3$. The quantum particle travels from $|0\rangle$ to $|1\rangle$. The $\langle n\rangle$ diverges when $U$ is close to the exceptional points. The weak charge envelope (blue solid line) clearly gives the tendency of the transition time. The theoretical result Eq. (54) (cyan solid line) fits quite well with the exact $\langle n\rangle$ (black dashed line). For $\langle n\rangle / V_{r}$, the exact result (red dashed line) gradually approaches to our theoretical value (green solid line), while $\langle n\rangle / V_{r} \leqslant 1 / 2$ in the whole regime. The fluctuations of the FDR give the upper bound of the corresponding mean FDT time in this example.

or to the site labeled $x=4$. Then the Hamiltonian of the ring reads

$$
H=-\gamma\left[\sum_{x=0}^{5}(|x\rangle\langle x+1|+| x+1\rangle\langle x|)\right] .
$$

We prepare our quantum particle in the state $|0\rangle$ and perform the detection in the state $|3\rangle$, which monitors the travel of the quantum particle from site 0 to the opposing site. In this case $P_{\text {det }}=1$ except for special sampling times.

The Hamiltonian of the benzene-type ring has the energy spectrum $E_{k}=-2 \cos \left(\theta_{k}\right)$ and the eigenstates are $\left|E_{k}\right\rangle^{T}=$ $\left(1, e^{i \theta_{k}}, e^{2 i \theta_{k}}, e^{3 i \theta_{k}}, e^{4 i \theta_{k}}, e^{5 i \theta_{k}}\right) / \sqrt{6}$ with $\theta_{k}=2 \pi k / 6$ and $k=$ $0,1,2,3,4,5$ (the superscript $T$ is the transpose). In this case we have four distinct energy levels so $w=4$. From Eqs. (22) and (23) the charges $p_{k}$ and $q_{k}$ read

$$
\begin{aligned}
& p_{1}=1 / 6, \quad p_{2}=1 / 6, \quad p_{3}=1 / 3, \quad p_{4}=1 / 3 ; \\
& q_{1}=1 / 6, \quad q_{2}=-1 / 6, \quad q_{3}=-1 / 3, \quad q_{4}=1 / 3 .
\end{aligned}
$$

As mentioned, the sampling time $\tau$ will introduce effective degeneracies to the problem. From Eq. (20), the exceptional sampling times are $\tau=\pi / 2,2 \pi / 3, \pi, 4 \pi / 3,2 \pi$ in the time interval $\{\tau \mid 0 \leqslant \tau \leqslant 2 \pi\}$. Close to these exceptional points we will have the scenario of two charges merging,

(i) When $\tau$ is close to $\pi / 2$ or $3 \pi / 2$ we have $\left|E_{1}-E_{2}\right| \tau \sim$ $2 \pi k$. The charges $p_{1}$ and $p_{2}$ coalesce [see Fig. 7(b)]. For the mean transition time $\langle n\rangle$ and $\langle n\rangle / V_{r}$, using Eqs. (47) and (50) we have

$$
\langle n\rangle \sim \frac{1}{36} \frac{1}{(\tau-\pi / 2)^{2}}, \quad \frac{\langle n\rangle}{V_{r}} \sim \frac{1}{6} .
$$

(ii) When $\tau$ is close to the $2 \pi / 3$ or $4 \pi / 3$ we have $\mid E_{1}-$ $E_{4} \mid \tau \sim 2 \pi k$ and $\left|E_{2}-E_{3}\right| \tau \sim 2 \pi k$. Two pairs of charges are merging separately [see Figs. 7(c) and 7(d)]. From Eqs. (47) and (50), due to the elimination $q_{1} p_{4}-q_{4} p_{1}=0$ and $q_{2} p_{3}-$ $q_{3} p_{2}=0$ we have

$$
\langle n\rangle \sim O(1), \quad\langle n\rangle / V_{r} \rightarrow 0 .
$$

The leading order of $\langle n\rangle$ vanishes, so $\langle n\rangle$ drops to some small values, leading to a small "discontinuity" on the graph. Close to these points we find that it takes less time for the walker to reach the detected state.

(iii) When $\tau$ is close to $\pi$ we also have two groups of charges merging Fig. 7(e), i.e., $p_{1}$ is close to $p_{2}$, and $p_{3}$ is close to $p_{4}$. Eq. (47) gives

$$
\langle n\rangle \sim \frac{1}{36} \frac{1}{(\tau-\pi)^{2}}+\frac{4}{9} \frac{1}{(\tau-\pi)^{2}} .
$$

For the ratio of $\langle n\rangle$ and $V_{r}$ there are two groups of charges which we treat separately. The first group we use Eq. (50) to obtain $\langle n\rangle_{1,2} / V_{1,2}=1 / 6$, where $\langle n\rangle_{1,2}$ and $V_{1,2}=1 / 6$ are the diagonal matrix term in Eqs. (38) and (39) related to the pole that is between the charge $p_{1}$ and $p_{2}$. Similarly, for the second group we have $\langle n\rangle_{3,4} / V_{3,4}=1 / 3$. The return variance $V_{r} \sim$ $V_{1,2}+V_{3,4}$ and the mean FDT time $\langle n\rangle \sim\langle n\rangle_{1,2}+\langle n\rangle_{3,4}=$ $V_{1,2} / 6+V_{3,4} / 3$. We can measure the fluctuations $V_{r}$ but not the terms $V_{1,2}$ and $V_{3,4}$. So here we do not have a direct relation between $\langle n\rangle$ and $V_{r}$. Using Eqs. (46) and (50), we first calculate $V_{1,2}$ and $V_{3,4}$ (then $V_{r} \sim V_{1,2}+V_{3,4}$ ). Comparing $V_{r}$ and Eq. (59), we have $\langle n\rangle / V_{r}=5 / 18$.

As shown in Fig. 7, we plot the exact results of $\langle n\rangle$ for $\tau$ from 0 to $2 \pi$. The theoretical predictions meet the exact values very well close to the exceptional points where the total detection probability exhibits a sudden jump in its value.

Our theoretical derivation of the Einstein like relation Eq. (42) is based on the approximation where a single pole $Z_{S}$ is approaching the unit disk. This leads to a relation between the variance of the FDR time and the mean of the FDT time for two cases: the single weak charge and merging two charges mechanisms, Eqs. (48) and (50) respectively. However, as we just showed, in some cases there are several poles approaching the unit disk together, i.e., part 3 of the Benzene-type ring. In generality the relation between fluctuations of the FDR time and the mean FDT time is more complicated in these cases, as the relevant expressions involve summations over several matrix elements, see Eqs. (38) and (39). We now treat a case where many poles $Z$ or the corresponding zeros $z=1 / Z^{*}$ approach the unit disk, a case we call big charge theory. Also this case gives large transition times, but technically it differs from the two previously studied cases of weak charge and merging charges. 


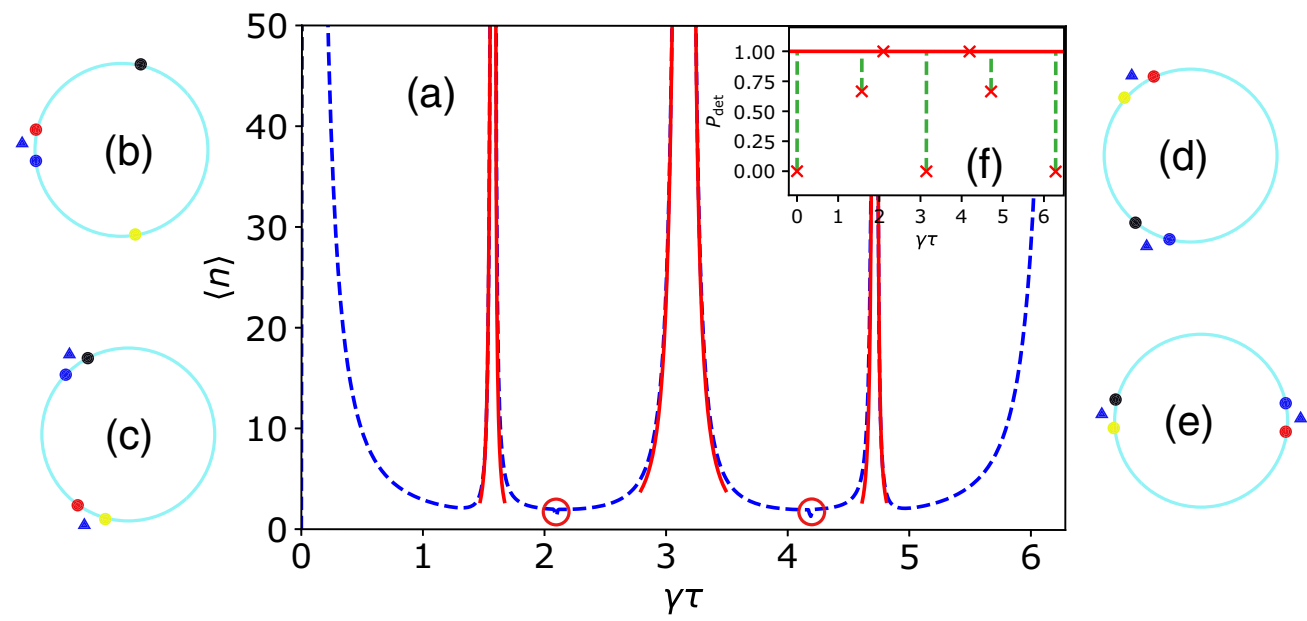

FIG. 7. The mean FDT time $\langle n\rangle$ versus $\gamma \tau$ of the Benzene-type ring (a). The quantum particle is prepared at $|0\rangle$ and the detector is set on the opposite site [see Fig. 4(c)]. When $\tau \rightarrow \pi / 2$ or $3 \pi / 2$, the charges $p_{1}$ (blue) and $p_{2}$ (red) come close to each other (b). The $P_{\text {det }}$ (f) drops to $2 / 3$, so both the two-charge theory Eq. (57) (red solid curve) and the exact result (blue dashed curve) diverge. When $\tau \rightarrow 2 \pi / 3$ or $4 \pi / 3$, there are two groups of charges merging (c) and (d). However because of the elimination of the resonance the leading term in the mean FDT time $\langle n\rangle$ vanishes [see Eqs. (47) and (58)] and it jumps to some small value instead of diverging as usual. The total detection probability remains unity at these special $\tau s$ (f). In the graph we have the "discontinuity" for $\langle n\rangle$ close to these points. On the unit disk, the blue point represents the charge $p_{1}$, the red is $p_{2}$, the yellow is $p_{3}$ and the black is $p_{4}$ [see subplots (b)-(e)]. In (b)-(e) which explain the charge picture, we choose $\tau$ according to (b) $\tau \rightarrow \pi / 2$ or $3 \pi / 2$, (c) $\tau \rightarrow 2 \pi / 3$, (d) $\tau \rightarrow 4 \pi / 3$, and (e) $\tau \rightarrow \pi$.

\section{BIG CHARGE THEORY}

Another scenario which leads to divergence of the mean FDT time $\langle n\rangle$ is when all the poles are close to the unit circle. This comes from the fact that the detected state is close to one of the eigenstates of Hamiltonian $H$, leading to a big charge appearing in the theory Eq. (23). Using Eq. (38), the off-diagonal terms in $\langle n\rangle$ are negligible compared with the diagonal terms, then we get

$$
\langle n\rangle \sim \sum_{i=0, i \neq b}^{w-1} \frac{\left|C_{i}\right|^{2}}{\left(-1+\left|Z_{i}\right|^{2}\right)^{2}}, \quad\left|Z_{i}\right| \sim 1 .
$$

The big charge, denoted $p_{b} \sim 1$, associated to the energy level $E_{b}$, is large in comparison with the other charges. Since the sum of all the charges is unity $\sum_{k=1}^{w-1} p_{k}=1$ and each of them is positive we have $1-p_{b}=\sum_{k \neq b} p_{k} \sim 0$. Hence there is one big charge $p_{b}$ and $w-1$ weak charges. Basic electrostatics indicates that the $w-1$ stationary points will lie close to the $w-1$ weak charges. From Eq. (29) we have $\left|Z_{i}\right|=1 /\left|z_{i}\right|$, such that all the poles $\left|Z_{i}\right| \rightarrow 1$ in this case. The charges and zeros of the problem are schematically shown in in Fig. 8. The $w$ charges have $w-1$ poles and all of them are close to the weak charges.

With the electrostatic analogy we obtain the zero force field as a balance between a weak charge and the strong one (on the line connecting these two charges). This is performed for each weak charge separately. Then this problem becomes a two-body problem (the charge $p_{b}$ and the weak charge $p_{i}$ ) for finding the stationary point between them, and all other charges are negligible. Using Eq. (30), the zeros are given by the root of $p_{b} /\left(e^{i E_{b} \tau}-z_{i}\right)+p_{i} /\left(e^{i E_{i} \tau}-z_{i}\right)=0$, which yields $z_{i}=\left(p_{i} e^{i E_{b} \tau}+p_{b} e^{i E_{i} \tau}\right) /\left(p_{i}+p_{b}\right)$. From the relation between zeros and poles in Eq. (29) we have $Z_{i} \sim e^{i E_{i} \tau}+\left[e^{i E_{i} \tau}-\right.$ $\left.e^{i\left(2 E_{i}-E_{b}\right) \tau}\right] p_{i} / p_{b}$. The $i$ goes from $i=0$ to $w-1$ but $i \neq b$, so all $w-1$ poles are found. The first part of $Z_{i}$ is just the location of the charge $p_{i}$, the second part is small and comes from the net field of $p_{i}$ and $p_{b}$. We put the $Z_{i}$ into Eq. (35) to get the coefficient $C_{i} \sim-q_{i} e^{i E_{i} \tau}\left[1-e^{i\left(E_{i}-E_{b}\right) \tau}\right] / p_{b}$. Here

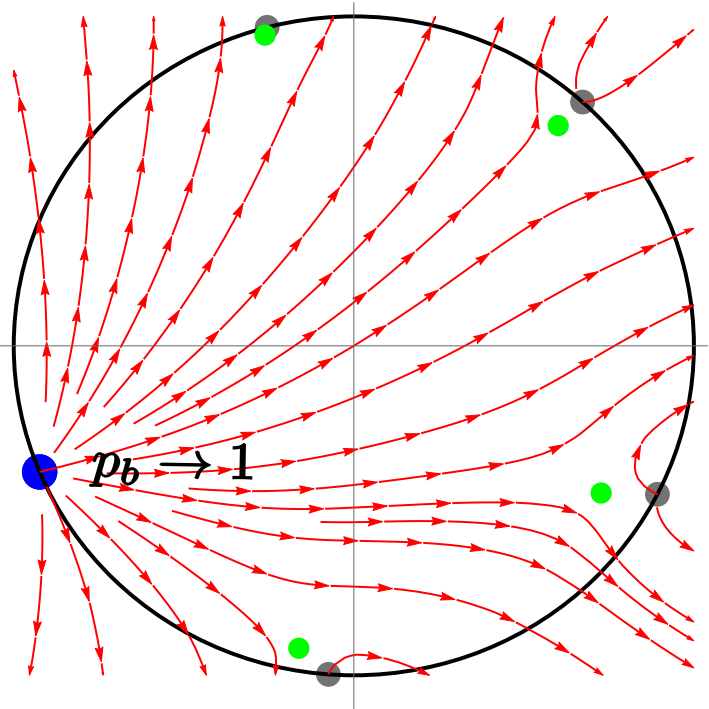

FIG. 8. Schematic plot of the zeros $\left\{z_{i}\right\}$ in the complex plane for the big charge theory. The red curves are the electric field lines, created by the charges on the unit circle. And this field vanishes at the green points which are the stationary points defined in Eq. (30). Here $p_{b} \rightarrow 1$ is the big charge and hence from normalization all other charges are weak. The stationary points ( $\left\{z_{i}\right\}$, green points) are close to the weak charges. As mentioned in the main text the poles $\left|Z_{i}\right|=1 /\left|z_{i}\right| \rightarrow 1$, and they are all out side the unit circle. 
enters the ratio of $q_{i}$ and the big charge $q_{i} / p_{b}$, which is a small parameter. $e^{i\left(E_{i}-E_{b}\right)}$ measures the phase difference between them. Substituting both $Z_{i}$ and $C_{i}$ into Eq. (35), the mean FDT time for the big charge scenario reads

$$
\langle n\rangle \sim \sum_{i=0, i \neq b}^{w-1} \frac{\left|q_{i}\right|^{2}}{4 p_{i}^{2} \sin ^{2}\left[\left(E_{i}-E_{b}\right) \tau / 2\right]} .
$$

It is interesting to recall that in our weak charge theory [see Eq. (45)] the envelope is given by $\left|q_{0}\right|^{2} / 4 p_{0}^{2}$, where $p_{0}$ is a weak charge. For the big charge we have $\left|q_{i}\right|^{2} / 4 p_{i}^{2}$, where $p_{i}$ is also small.

\section{Localized wave function}

A nice example for the big charge theory is when the quantum particle is effectively localized at the detected state by a strong potential (but the initial state $\left|\psi_{\text {in }}\right\rangle$ is not at the state of the potential). To establish a specific example, we choose a five-site linear molecule put the detector at the site $x=0$ and prepare the initial state at $|4\rangle$. We add a potential barrier $U$ at site $x=0$ [see Fig. 4(d)]. Then the Hamiltonian of this five-site molecule reads

$$
H=-\gamma\left[\sum_{x=0}^{4}(|x\rangle\langle x+1|+| x+1\rangle\langle x|)+U|0\rangle\langle 0|\right] .
$$

Here the boundary conditions are that from the site labeled $x=4$ one can only hop to the site labeled $x=3$, and from the site labeled $x=0$ one can only hop to the site labeled $x=1$.

For the energy spectrum we consider the regime where the wave function is effectively localized. As we increase the value of $U$, the energy level $E_{0} \rightarrow-U$. At the same time, this large potential well makes it difficult for the quantum particle to hop to the state $|0\rangle$. So the remaining four energy levels are given by the new Hamiltonian $H_{l}=-\gamma \sum_{x=1}^{4}(|x\rangle\langle x+1|+$ $|x+1\rangle\langle x|)$ with the same boundary condition as Eq. (62). Hence the energy spectrum reads $E_{0} \sim-U, E_{1} \sim(1+$ $\sqrt{5}) / 2, E_{2} \sim-(1+\sqrt{5}) / 2, E_{3} \sim(-1+\sqrt{5}) / 2$, and $E_{4} \sim$ $(1-\sqrt{5}) / 2$. Notice that the energy levels are nondegenerate hence $w=5$. The exact values of the energy levels are calculated and depicted in Appendix A in Fig. 10(c).

Next we prepare the quantum particle in the state $|4\rangle$, such that the system describes the movement of the particle from site $x=4$ to $x=0$ on a linear molecule. From Eq. (23) it follows that the big charge $p_{0} \rightarrow 1$ and the remaining weak charges $p_{i \neq 0} \rightarrow 0$. With Eq. (61) we get the mean FDT time

$$
\langle n\rangle \sim \sum_{i=1}^{4} \frac{\left|q_{i}\right|^{2}}{4 p_{i}^{2} \sin ^{2}\left[\left(E_{i}-E_{0}\right) \tau / 2\right]} .
$$

In Fig. 9 we compare the numerical result with our big charge theory, choosing the sampling time $\tau=1$ and the potential well goes from 0 to 15 . In the limit of large $U$ the four weak charges are fixed on the unit circle, their positions are given by their $U$-independent phase $\exp \left(i E_{i} \tau\right)$. When we increase $U$ the strong charge $p_{0}$, is thus crossing the location of the other charges and in the range $0<U<15$ which happens twice $(15 / 2 \pi \sim 2)$. As shown in Fig. 9, we have two groups of divergencies each with four peaks. The number of peaks in each group is $w-1=4$.

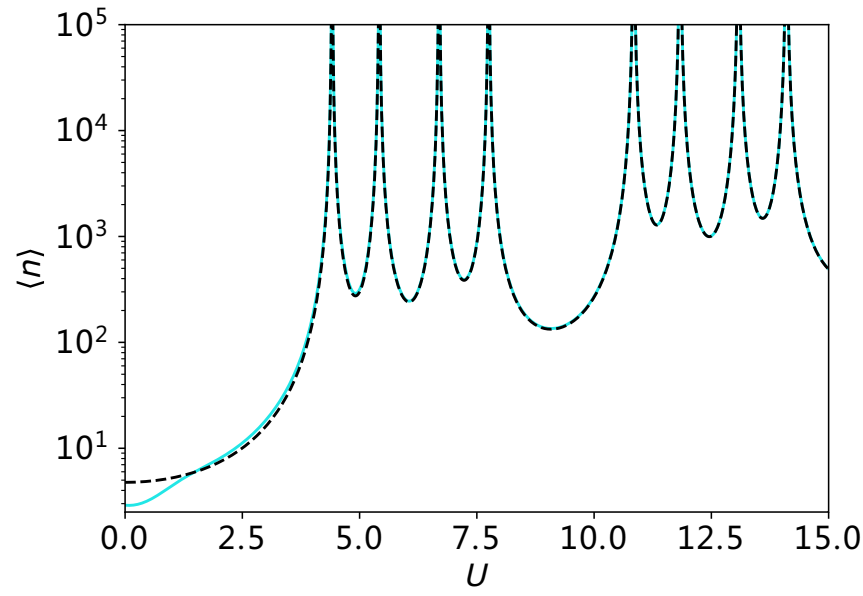

FIG. 9. The mean FDT time $\langle n\rangle$ versus $U$ for the five-site molecule. Here the sampling time is $\tau=1$ and the quantum particle moves from $|4\rangle$ to $|0\rangle$, see Fig. 4(d). The exact values (black dashed line) are calculated from Eq. (38). The cyan line is our big charge theory result Eq. (63). Close to the exceptional points given by Eq. (20), the mean FDT times diverge. Since the strong charge $p_{0}$ rotates two laps on the unite circle, there are two clusters of peaks. In each cluster the big charge passes through the remaining four charges, leading to the four peaks in the graph.

\section{DISCUSSION AND CONCLUSIONS}

We investigated the mean FDT time for closed quantum systems in a finite-dimensional Hilbert space, where repeated measurements interrupt the unitary evolution by a projection after a time step $\tau$. This has a strong effect on the dynamical properties. A general formula Eq. (38) for the mean FDT time was derived, which answers how long it takes to find a particle in a certain state under repeated measurements. The unitary evolution $\exp (-i H \tau)$ is controlled by the energy spectrum, and the overlaps $\left\{p_{k}\right\}$ and $\left\{q_{k}\right\}$ in Eqs. (22) and (23) which are crucial in that they connect the eigenstates of the Hamiltonian $H$ with the initial and measured states. Moreover, the nonunitary evolution is characterized by the zeros of the polynomial Eq. (27) and the overlap functions. Those zeros are formally related to a classical electrostatic problem [13]; namely they are the stationary points of a test charge in a system with charges on the unit circle, which is defined in Eq. (30).

After solving this electrostatic problem, the zeros are used to calculate, for instance, the first detection amplitude in Eq. (36) and the divergent behavior of the mean FDT time near degenerate points in Eq. (38). The charge theory provides an intuitive picture to gain physical insight, in particular, when zeros approach the unit circle. Then we focused on the diverging mean FDT times, where the total detection probability exhibits a discontinuous drop of its value. This property is nonclassical and important for the search problem.

When the mean FDT time diverges, we find a relation between the latter and the variance of the FDR time, Eq. (42). This relation is reminiscent of the Einstein relation (or the fluctuation-dissipation theorem) in classical statistical physics. In contrast to the latter, where thermal fluctuations are considered, in our case the quantum fluctuations are 
described by the variance of the FDR time. Another point worthwhile to mention is that our relation between the FDT time and the variance of the FDR time is valid only when the system is close to the critical parameter values. Hence this Einstein like relation indicates a universal connection between the FDT time and FDR fluctuations only near critical parameters. More technically this means that a single pole is approaching the unit disk. Such cases are treated with the weak charge and merging charges mechanisms, and these lead to explicit Einstein like relation Eqs. (48) and (50). The case when more than one pole is approaching the unit disk was treated with the single big charge theory. Also in this case the mean FDT time diverges, and so does the variance of the return. However since we need to add up many contributions the Einstein like relation is expected to have a tensor form, with details left for future work. In this case a measurement of the FDR time variance cannot be used to predict directly the mean FDT time, but rather one needs to calculate the different contributions to $V_{r}$ and $\langle n\rangle$, see Eqs. (38) and (39).

Previous work has indicated the advantage of repeated monitored search [33-36], in particular, Krovi and Brun $[10,12]$ investigated the quantum search on the hyper cube, where constructive interference can bring a dramatic increase in the speed of search under repeated measurement. However, the speed up depends on specific initial conditions, and hence in generality quantum search is not always efficient, as other initial conditions can lead to a slow down. All these situations are covered by our general formula for the transition time. This can be used to optimize the time of search. On the other hand, it can be used to understand and avoid a slow down of the search. In this paper we discuss the role of spectral degeneracies and the role of vanishing spectral weights for a diverging search time. More work is needed for the analysis of more complex examples, such as larger systems with one particle and many-body systems.

There are still many open questions in this field. For example, if we apply weak measurements in this process rather than the strong collapse measurements, what is the mean FDR or FDT time? Also, if the waiting time between two measurements is not constant, for instance, Poisson distributed, what will we get? Does the diverging FDT time still exist? As we mentioned before, this kind of research is not only addressing the detection time in a closed quantum system but also relevant to basic physics and possibly contributes to the quantum search problem. For example, the zeros $\left\{z_{i}\right\}$ investigated here are important not only for the first detection problem, but rather they control different dynamical aspects of monitored quantum dynamics, like quantum control via measurement.

\section{ACKNOWLEDGMENTS}

We thank Felix Thiel and David Kessler, for many helpful discussions, which led to simplifications of some of the formulas of this paper. The support of Israel Science Foundation's Grant No. 1898/17 as well as the support by the Julian Schwinger Foundation (K.Z.) are acknowledged.

\section{APPENDIX A: ORDER OF $\mathcal{G}(z)$ AND $\mathcal{D}(z)$}

In this section we proof $\operatorname{deg}[\mathcal{D}(z)]>\operatorname{deg}[\mathcal{G}(z)]$ used in the main text. Since $\mathcal{G}(z) \simeq \sum q_{i} z^{w-1}+\cdots$, the highest order of $\mathcal{G}(z)$ is $\left(\sum_{i=0}^{w-1} q_{i}\right) z^{w-1}$. However, what is special in the transition problem is $\sum_{i=0}^{w-1} q_{i}=\left\langle\psi_{\mathrm{d}} \mid \psi_{\text {in }}\right\rangle=0$, namely that the highest order vanishes, such that $\operatorname{deg}(\mathcal{G})<w-1$ for the numerator.

Using Eq. (27), $\mathcal{D}(z) \simeq \sum p_{i} e^{i E_{i} \tau} z^{w-1}+\cdots$ the leading order of $z$ is $\beta z^{w-1}=\left(\sum_{i=0}^{w-1} p_{i} e^{i E_{i} \tau}\right) z^{w-1}$.

$$
\sum_{i=0}^{w-1} p_{i} e^{i E_{i} \tau}=\left\langle\psi_{\mathrm{d}}\left|e^{i \hat{H} \tau}\right| \psi_{\mathrm{d}}\right\rangle \neq 0 .
$$

Hence $\operatorname{deg}[\mathcal{D}(z)]>\operatorname{deg}[\mathcal{G}(z)]$.

The energy levels versus the potential $U$ is shown in Fig. 10.

\section{APPENDIX B: WEAK CHARGE}

In this section we derive Eqs. (44) and (45) of the main text. Following the same procedure, we can derive Eqs. (47) in the Sec. VI.

As we mentioned in the main text, the weak charge $p_{0} \sim 0$ and the corresponding energy level is $E_{0}$. Using Eq. (30), we have:

$$
0=\sum_{k=0}^{w-1} \frac{p_{k}}{e^{i \tau E_{k}}-z}=\frac{p_{0}}{e^{i \tau E_{0}}-z}+\sum_{k=1}^{w-1} \frac{p_{k}}{e^{i \tau E_{k}}-z} .
$$

Assuming that $z_{0}=e^{i E_{0} \tau}-\epsilon$. The $\epsilon$ is the first-order approximation. Using Eq. (B1), we have:

$$
0 \approx \frac{p_{0}}{\epsilon}+\sum_{k=1}^{w-1} \frac{p_{k}}{e^{i \tau E_{k}}-e^{i \tau E_{1}}},
$$

and hence

$$
\epsilon \sim \frac{p_{0}}{\sum_{k=1}^{w-1} p_{k} /\left(e^{i \tau E_{1}}-e^{i \tau E_{k}}\right)} .
$$

Using Eq. (29), the pole $Z_{0}$ in the main text reads:

$$
Z_{0}=\frac{1}{z_{0}^{*}}=\frac{1}{e^{-i \tau E_{0}}-\epsilon^{*}} \sim e^{i \tau E_{0}}\left(1+\epsilon^{*} e^{i \tau E_{0}}\right) .
$$

The index $C_{i}$ is defined in Eq. (35). Plugging the pole $Z_{0}$ into Eq. (35), we obtain

$$
\begin{aligned}
\mathcal{N}\left(Z_{0}\right) & \sim-e^{i E_{0} \tau}\left[\frac{q_{0}}{-\epsilon^{*} e^{2 i E_{0} \tau}}+\sum_{j=1}^{w-1} \frac{q_{j}}{\left(e^{i E_{j} \tau}-e^{i E_{0} \tau}\right)}\right] \\
& \sim \frac{q_{0}}{\epsilon^{*} e^{i E_{0} \tau}}
\end{aligned}
$$

and

$$
\begin{aligned}
\mathcal{D}^{\prime}\left(Z_{0}\right) & \sim \frac{p_{0} e^{i E_{0} \tau}}{\epsilon^{* 2} e^{4 i E_{0} \tau}}+\sum_{j=1}^{w-1} \frac{p_{j} e^{i E_{j} \tau}}{\left(e^{i E_{j} \tau}-e^{i E_{0} \tau}\right)^{2}} \\
& \sim \frac{p_{0}}{\epsilon^{* 2} e^{3 i E_{0} \tau}} .
\end{aligned}
$$



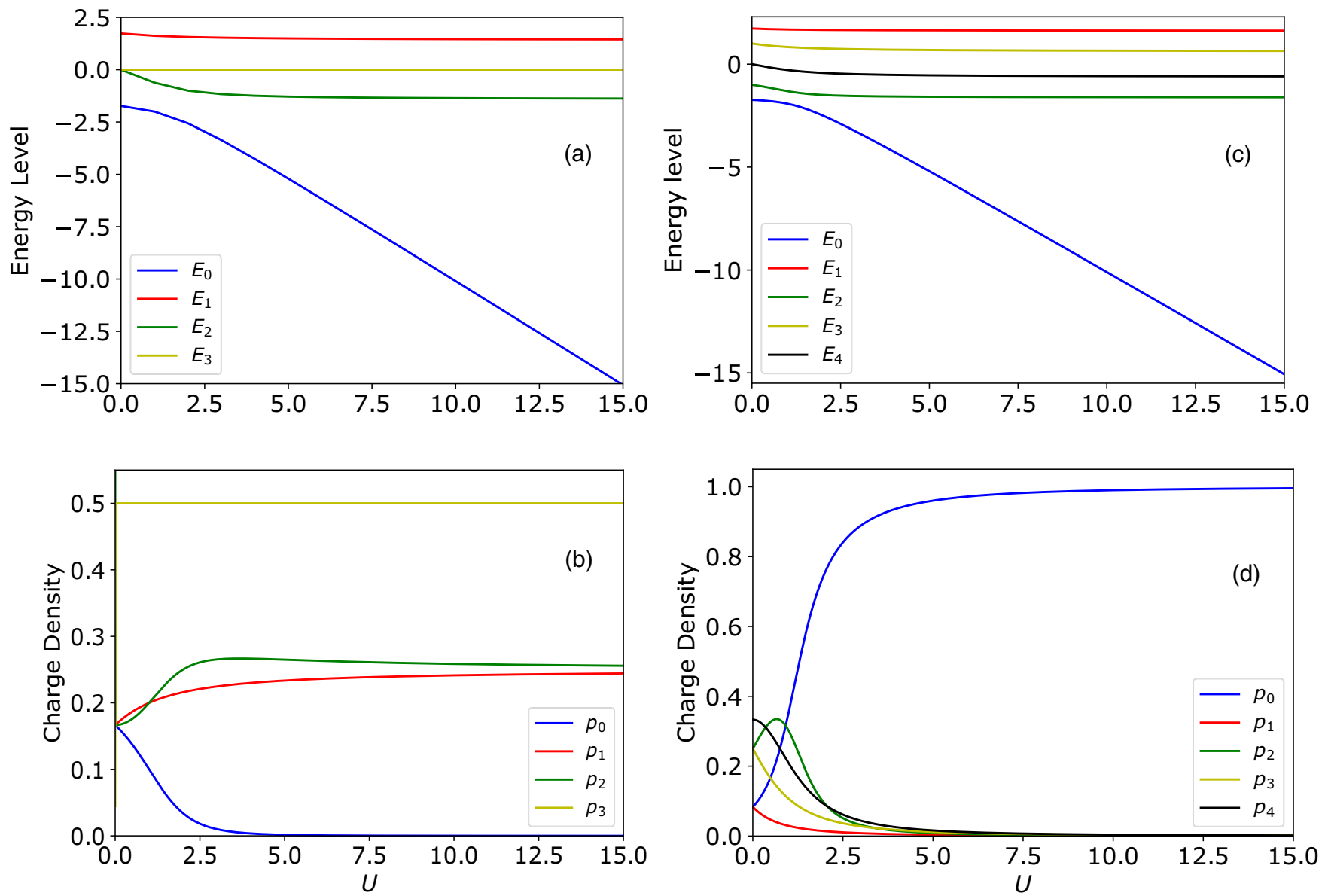

FIG. 10. (a) The energy levels versus the potential $U$ of the Y-shaped molecule. (b) The energy levels versus the potential $U$ of the linear five-site molecule. (c) The charges $p_{k}$ versus the potential $U$ of the $\mathrm{Y}$-shaped molecule. (d) The charges $p_{k}$ versus the potential $U$ of the linear five-site molecule.

Hence the coefficient $C_{0}$ used in the main text reads:

$$
C_{0} \sim \frac{q_{0}}{p_{0}} \epsilon^{*} e^{2 i E_{0} \tau} .
$$

Substituting $C_{0}$ and $Z_{0}$ into Eq. (43), the mean FDT time becomes

$$
\langle n\rangle \sim \frac{\left|C_{0}\right|^{2}}{\left(\left|Z_{0}\right|^{2}-1\right)^{2}} \sim \frac{\left|q_{0}\right|^{2}}{p_{0}^{2}} \frac{|\epsilon|^{2}}{\left(2 \operatorname{Re}\left[\epsilon * e^{-i E_{0} \tau}\right]\right)^{2}} .
$$

Using the mathematical property $1 /(1-\exp [i x])=1 / 2+$ $i \cot [x / 2] / 2$ and the normalization condition $\sum_{k=1}^{w-1} p_{k}=1-$ $p_{0} \sim 1$, we can simplify the parameter $\epsilon$. From Eq. (B3), we have:

$$
\begin{aligned}
\epsilon & \sim \frac{p_{0}}{\sum_{k=1}^{w-1} p_{k} /\left(e^{i \tau E_{0}}-e^{i \tau E_{k}}\right)} \\
& =e^{i E_{0} \tau} \frac{p_{0}}{\sum_{k=1}^{w-1} p_{k} /\left(1-e^{i \tau\left(E_{k}-E_{0}\right)}\right)} \\
& =e^{i E_{0} \tau} \frac{2 p_{0}}{\sum_{k=1}^{w-1} p_{k}\left(1+i \cot \left[\tau\left(E_{k}-E_{0}\right) / 2\right]\right)} \\
& \sim e^{i E_{0} \tau} \frac{2 p_{0}}{1+i \sum_{k=1}^{w-1} p_{k} \cot \left[\tau\left(E_{k}-E_{0}\right) / 2\right]} .
\end{aligned}
$$

Plugging $\epsilon$ into Eq. (B6), the mean FDT time reads

$$
\langle n\rangle \sim \frac{\left|q_{0}\right|^{2}}{4 p_{0}^{2}}\left(1+\left\{\sum_{k=1}^{w-1} p_{k} \cot \left[\left(E_{k}-E_{0}\right) \tau / 2\right]\right\}^{2}\right) .
$$

\section{APPENDIX C: TWO-CHARGE POLE $Z_{p}$}

In this section we derive $Z_{p}$ of the main text. When a pair of charges is nearly merging, say $\exp \left(i E_{a} \tau\right) \simeq \exp \left(i E_{b} \tau\right)$, one of the zeros denoted $z_{p}$ will be close to the unit circle. We define $2 \delta=\left(\bar{E}_{b}-\bar{E}_{a}\right) \tau$, hence $\delta$ is a order parameter measuring this process. We first consider the two merging charges. Using Eq. (30) we have

$$
\frac{p_{a}}{e^{i E_{a} \tau}-z}=-\frac{p_{b}}{e^{i E_{b} \tau}-z}
$$

which yields

$$
z_{p}^{(0)}=\frac{p_{a} e^{i E_{b} \tau}+p_{b} e^{i E_{a} \tau}}{p_{a}+p_{b}} .
$$

Now we take the background charges into consideration.

$$
z_{p}=z_{p}^{(0)}-z_{p}^{(1)} .
$$


Plugging $z_{p}$ into Eq. (30), we have

$$
\begin{aligned}
0= & \sum_{k=0}^{w-1} \frac{p_{k}}{e^{i E_{k} \tau}-z} \\
\approx & \frac{p_{a}}{e^{i E_{a} \tau}-z_{p}^{(0)}+z_{p}^{(1)}}+\frac{p_{b}}{e^{i E_{b} \tau}-z_{p}^{(0)}+z_{p}^{(1)}} \\
& +\sum_{k \neq a, b}^{w-1} \frac{p_{k}}{e^{i E_{k} \tau}-z_{p}^{(0)}} .
\end{aligned}
$$

The third part on the right-hand side is the effect of the background charges. We define it as $B$.

$$
B=\sum_{k \neq a, b}^{w-1} \frac{p_{k}}{e^{i E_{k} \tau}-z_{p}^{(0)}} \approx \sum_{k \neq a, b}^{w-1} \frac{p_{k}}{e^{i E_{k} \tau}-e^{i \tau \frac{E_{A}+E_{B}}{2}}} .
$$

Using Eqs. (C4) and (C5), we obtain

$$
\begin{aligned}
z_{p}^{(1)} & \sim \frac{B p_{a} p_{b}\left(e^{i E_{a} \tau}-e^{i E_{b} \tau}\right)^{2}}{\left(p_{a}+p_{b}\right)^{3}+B\left(p_{a}^{2}-p_{b}^{2}\right)\left(e^{i E_{a} \tau}-e^{i E_{b} \tau}\right)} \\
& \sim \frac{B p_{a} p_{b}\left(e^{i E_{a} \tau}-e^{i E_{b} \tau}\right)^{2}}{\left(p_{a}+p_{b}\right)^{3}} .
\end{aligned}
$$

Since $e^{i E_{B} \tau}-e^{i E_{A} \tau} \sim \delta, z_{p}^{(1)} \sim \delta^{2}$. The background charges give only a second-order effect $O\left(\delta^{2}\right)$ to the zero $z_{p}$ as we expected. Using Eq. (29) we have

$$
\begin{aligned}
Z_{p}= & Z_{p}^{(0)}+Z_{p}^{(1)}=\frac{1}{z_{p}^{*}} \\
\approx & \frac{p_{A}+p_{B}}{p_{A} e^{-i E_{B} \tau}+p_{B} e^{-i E_{A} \tau}} \\
& +\frac{B^{*} p_{A} p_{B}\left(e^{-i E_{A} \tau}-e^{-i E_{B} \tau}\right)^{2}}{\left(p_{A}+p_{B}\right)\left(p_{A} e^{-i E_{B} \tau}+p_{B} e^{-i E_{A} \tau}\right)^{2}} .
\end{aligned}
$$

[1] S. Redner, A Guide to First-Passage Processes (Cambridge University Press, Cambridge, UK, 2001).

[2] R. Metzler, G. Oshanin, and S. Redner, First-Passage Phenomena and Their Applications (World Scientific, Singapore, 2014).

[3] Y. Aharonov, L. Davidovich, and N. Zagury, Quantum random walks, Phys. Rev. A 48, 1687 (1993).

[4] E. Farhi and S. Gutmann, Quantum computation and decision trees, Phys. Rev. A 58, 915 (1998).

[5] A. M. Childs, E. Farhi, and S. Gutmann, An example of the difference between quantum and classical random walks, Quant. Inf. Process 1, 35 (2002).

[6] M. Karski, L. Förster, J. Choi, A. Steffen, W. Alt, D. Meschede, and A. Widera, Quantum walk in position space with single optically trapped atoms, Science 325, 174 (2009).

[7] F. Zähringer, G. Kirchmair, R. Gerritsma, E. Solano, R. Blatt, and C. F. Roos, Realization of a Quantum Walk with One and Two Trapped Ions, Phys. Rev. Lett. 104, 100503 (2010).

[8] P. M. Preiss, R. Ma, M. E. Tai, A. Lukin, M. Rispoli, P. Zupancic, Y. Lahini, R. Islam, and M. Greiner, Strongly correlated quantum walks in optical lattices, Science 347, 1229 (2015).

[9] E. Bach, S. Coppersmith, M. P. Goldschen, R. Joynt, and J. Watrous, One-dimensional quantum walks with absorbing boundaries, J. Comput. Syst. Sci. 69, 562 (2004).

[10] H. Krovi and T. A. Brun, Hitting time for quantum walks on the hypercube, Phys. Rev. A 73, 032341 (2006).

[11] H. Krovi and T. A. Brun, Quantum walks with infinite hitting times, Phys. Rev. A 74, 042334 (2006).

[12] M. Varbanov, H. Krovi, and T. A. Brun, Hitting time for the continuous quantum walk, Phys. Rev. A 78, 022324 (2008).

[13] F. A. Grünbaum, L. Velázquez, A. H. Werner, and R. F. Werner, Recurrence for discrete time unitary evolutions, Commun. Math. Phys. 320, 543 (2013).

[14] J. Bourgain, F. A. Grünbaum, L. Velázquez, and J. Wilkening, Quantum recurrence of a subspace and operator-valued schur functions, Commun. Math. Phys. 329, 1031 (2014).
[15] P. L. Krapivsky, J. M. Luck, and K. Mallick, Survival of classical and quantum particles in the presence of traps, J. Stat. Phys. 154, 1430 (2014).

[16] S. Dhar, S. Dasgupta, A. Dhar, and D. Sen, Detection of a quantum particle on a lattice under repeated projective measurements, Phys. Rev. A 91, 062115 (2015).

[17] S. Dhar, S. Dasgupta, and A. Dhar, Quantum time of arrival distribution in a simple lattice model, J. Phys. A: Math. Theor. 48, 115304 (2015).

[18] H. Friedman, D. A. Kessler, and E. Barkai, Quantum walks: The first detected passage time problem, Phys. Rev. E 95, 032141 (2017).

[19] F. Thiel, E. Barkai, and D. A. Kessler, First Detected Arrival of a Quantum Walker on an Infinite Line, Phys. Rev. Lett. 120, 040502 (2018).

[20] P. Kuklinski, Conditional probability distributions of finite absorbing quantum walks, Phys. Rev. A 101, 032309 (2020).

[21] B. Mukherjee, K. Sengupta, and S. N. Majumdar, Quantum dynamics with stochastic reset, Phys. Rev. B 98, 104309 (2018).

[22] D. C. Rose, H. Touchette, I. Lesanovsky, and J. P. Garrahan, Spectral properties of simple classical and quantum reset processes, Phys. Rev. E 98, 022129 (2018).

[23] S. Belan and V. Parfenyev, Optimal measurement protocols in quantum zeno effect, arXiv:1909.03226.

[24] S. Gherardini, Exact nonequilibrium quantum observable statistics: A large-deviation approach, Phys. Rev. A 99, 062105 (2019).

[25] D. Ben-Zion, J. McGreevy, and T. Grover, Disentangling quantum matter with measurements, Phys. Rev. B 101, 115131 (2020).

[26] A. Zabalo, M. J. Gullans, J. H. Wilson, S. Gopalakrishnan, D. A. Huse, and J. H. Pixley, Critical properties of the measurementinduced transition in random quantum circuits, Phys. Rev. B 101, 060301 (2020).

[27] B. Skinner, J. Ruhman, and A. Nahum, Measurement-Induced Phase Transitions in the Dynamics of Entanglement, Phys. Rev. X 9, 031009 (2019). 
[28] S. Roy, J. T. Chalker, I. V. Gornyi, and Y. Gefen, Measurementinduced steering of quantum systems, arXiv:1912.04292.

[29] L. P. Kouwenhoven, D. G. Austing, and S. Tarucha, Few-electron quantum dots, Rep. Prog. Phys. 64, 701 (2001).

[30] C. Zipkes, S. Palzer, L. Ratschbacher, C. Sias, and M. Köhl, Cold Heteronuclear Atom-Ion Collisions, Phys. Rev. Lett. 105, 133201 (2010).

[31] J. F. Sherson, C. Weitenberg, M. Endres, M. Cheneau, I. Bloch, and S. Kuhr, Single-atom-resolved fluorescence imaging of an atomic mott insulator, Nature 467, 68 (2010).

[32] J. T. Muhonen, J. P. Dehollain, A. Laucht, S. Simmons, R. Kalra, F. E. Hudson, A. S. Dzurak, A. Morello, D. N. Jamieson, J. C. McCallum, and K. M. Itoh, Coherent control via weak measurements in ${ }^{31} \mathrm{P}$ single-atom electron and nuclear spin qubits, Phys. Rev. B 98, 155201 (2018).

[33] L. K. Grover, Quantum Mechanics Helps in Searching for a Needle in a Haystack, Phys. Rev. Lett. 79, 325 (1997).

[34] A. M. Childs and J. Goldstone, Spatial search by quantum walk, Phys. Rev. A 70, 022314 (2004).

[35] J. Kempe, Discrete quantum walks hit exponentially faster, Probab. Theory Relat. Fields 133, 215 (2005).

[36] S. Chakraborty, L. Novo, A. Ambainis, and Y. Omar, Spatial Search by Quantum Walk is Optimal for Almost All Graphs, Phys. Rev. Lett. 116, 100501 (2016).
[37] R. Yin, K. Ziegler, F. Thiel, and E. Barkai, Large fluctuations of the first detected quantum return time, Phys. Rev. Res. 1, 033086 (2019).

[38] B. Misra and E. C. G. Sudarshan, The zeno's paradox in quantum theory, J. Math. Phys. 18, 756 (1977).

[39] M. Štefaňák, I. Jex, and T. Kiss, Recurrence and Pólya Number of Quantum Walks, Phys. Rev. Lett. 100, 020501 (2008).

[40] P. Xue, R. Zhang, H. Qin, X. Zhan, Z. H. Bian, J. Li, and B. C. Sanders, Experimental Quantum-Walk Revival with a Time-Dependent Coin, Phys. Rev. Lett. 114, 140502 (2015).

[41] F. Thiel, I. Mualem, D. A. Kessler, and E. Barkai, Uncertainty and symmetry bounds for the quantum total detection probability, Phys. Rev. Research 2, 023392 (2020).

[42] F. Thiel, I. Mualem, D. Meidan, E. Barkai, and D. A. Kessler, Quantum total detection probability from repeated measurements I. the bright and dark states, arXiv:1906.08112.

[43] S. Denisov, T. Laptyeva, W. Tarnowski, D. Chruściński, and K. Życzkowski, Universal Spectra of Random Lindblad Operators, Phys. Rev. Lett. 123, 140403 (2019).

[44] L. Sá, P. Ribeiro, and T. Prosen, Complex Spacing Ratios: A Signature of Dissipative Quantum Chaos, Phys. Rev. X 10, 021019 (2020).

[45] F. Thiel, I. Mualem, D. A. Kessler, and E. Barkai, Quantum total detection probability from repeated measurements II. exploiting symmetry, arXiv:1909.02114. 